Published as:

Rogers, L.; Burke-Murphy, E.; Senge, M. O. (2014):

Simple Porphyrin Desymmetrization: mTHPP as a Gateway Molecule for Peripheral Functionalization.

European Journal of Organic Chemistry, 4283-4294.

doi: http://dx.doi.org/10.1002/ejoc.201402433

\title{
Simple Porphyrin Desymmetrization: THPP as a Gateway Molecule for Peripheral Functionalization
}

\author{
Luke Rogers, ${ }^{[\mathrm{a}]}$ Emeralda Burke-Murphy, ${ }^{[\mathrm{a}]}$ Mathias O. Senge, ${ }^{[\mathrm{a}]}$
}

\begin{abstract}
The rise in the demand towards unsymmetric porphyrin systems for use in applications such as photodynamic therapy and non-linear optics mandates a concomitant development of simple and practical syntheses thereof. A new methodology for the rapid generation of unsymmetrical porphyrins synthesized from the same common and easily accessible symmetric porphyrin starting material is discussed herein. A library of unsymmetric $m$ THPP $[5,10,15,20$ tetrakis(3-hydroxyphenyl)porphyrin]
\end{abstract}

\section{Introduction}

Since their discovery by Rothemund in 1935, symmetric meso-aryl substituted porphyrins have been the workhorses of porphyrin chemistry, as they can be easily prepared in high yielding one pot reactions on a multi-gram scale. ${ }^{[1]}$ However, most applications such as photodynamic therapy ${ }^{[2]}$ (PDT), non-linear optics (NLO) ${ }^{[3]}$ solarenergy conversion ${ }^{[4]}$ and photosynthetic reaction center mimics ${ }^{[5]}$ call for unsymmetric porphyrin derivatives, e.g., amphiphilic systems for PDT or push-pull systems for energy transfer and optical studies. The last decades have seen vast improvements in the synthesis of unsymmetrical porphyrins either through a total synthesis approach, ${ }^{[6]}[2+2]$ or $[3+1]$ condensation reactions, or via functionalization reactions. Especially the last two, exemplified by Lindsey's large scale syntheses ${ }^{[6 b-d]}$ and our functionalization approach for ABCD-type porphyrins ${ }^{[7]}$ amongst others have found wide spread use in the synthetic community. Drawbacks of this

[a] L. Rogers, E. Burke-Murphy, Prof. Dr. M. O. Senge SFI Tetrapyrrole Laboratory, School of Chemistry Trinity Biomedical Sciences Institute 152-160 Pearse Street Trinity College Dublin Dublin 2 (Ireland) Fax: (+) 353-1-896-8536 E-mail: sengem@tcd.ie fence porphyrin mimics and gave such compounds in good yields and few synthetic steps. Likewise, employing simple nucleophilic substitution chemistry co-facial, 'caged' bisporphyrin systems were accessible in two synthetic steps.

Keywords: porphyrinoids • nucleophilic substitution • tetrapyrroles $\bullet$ macrocycles $\bullet$ photodynamic therapy 
the plethora of unsymmetrical porphyrins necessary today Nowadays, the porphyrin field is driven by application oriented research with significant contributions coming from material science, inorganic, physical and medicinal chemists who lack the synthetic capabilities necessary for the more involved syntheses. Thus, there is a pressing need for simple, technically facile syntheses for unsymmetrically substituted porphyrins. We here report one such approach, which relies on the monofunctionalization of symmetric $\mathrm{A}_{4}$-porphyrins using simple substitution chemistry (Fig. 1).

\section{Results and Discussion}

Synthetic concept and background: Our approach emerged from our interest in advancing the photodynamic cancer therapy (PDT) of esophageal cancer through the synthesis of dual modality photosensitizers (PS) ${ }^{[9]}$ In this context we wanted to conjugate a mono-alkyne porphyrin to a bile acid azide through a click reaction to form a library of potential photosensitizers. Instead of embarking on a long synthesis using methods mentioned previously to synthesize a mono-alkynyl porphyrin, we decided to use a known porphyrin PS as the core scaffold upon which we could conjugate a number of different biologically active bile acids. ${ }^{[10]}$ The immediately appealing candidate was 5,10,15,20-tetrakis(3hydroxyphenyl)chlorin ( $m$ THPC, 1), an approved second generation PS. ${ }^{[11]}$ For synthetic ease we first focused on 5,10,15,20-tetrakis(3tetrahydroxyphenyl)porphyrin ( $m$ THPP, 2), the parent compound to $m$ THPC, which is a highly soluble symmetric porphyrin and also a potent photosensitizer. ${ }^{[11 c]}$

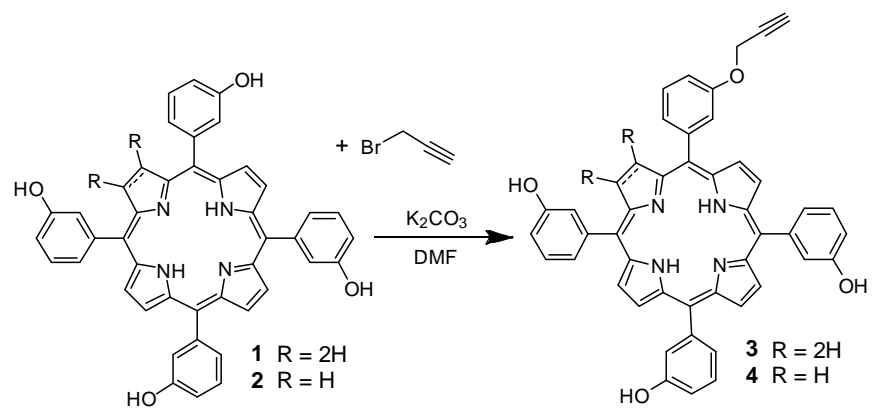

Scheme 1. Monofunctionalization of $m$ THPC (1) and $m$ THPP (2).

Interestingly, little research has been conducted into the controlled functionalization of tetrasubstituted hydroxyporphyrins which can be synthesized in multi-gram quantities. Our plan was to use 2 as a preformed porphyrin scaffold through which we can selectively install a variety of functional groups through cheap, simple and reliable chemistry. Once a library was generated, we then aimed to synthesize a number of unsymmetrical porphyrins tailored for a variety of applications, in particular PDT.

The starting point of the synthesis began with the requirement of an alkyne source which was amenable to nucleophilic substitution reactions, and we first chose propargyl bromide. The first difficulty encountered was the four equivalent phenol groups present in $m$ THPP and whether we could maximize the yield of monosubstituted product whilst limiting the production of higher order reaction products. Taking compound 2 and reacting it with 5 eq. of propargyl bromide for $5 \mathrm{~h}$, in the presence of $\mathrm{K}_{2} \mathrm{CO}_{3}$, we were successful in selectively mono-functionalizing this porphyrin in $18 \%$ yield (Scheme 1). The alkynyl residue in 4 then served as a synthetic handle upon which we could use a modified Huisgen cycloaddition reaction $^{[12 \mathrm{a}]}$ between a bile acid azide and, in this case, a terminal alkyne to give a $1,2,3$-triazole. ${ }^{[12 b]}$

Optimization: Whilst a yield of $18 \%$ was sufficient for obtaining enough material for the necessary click reaction above, we next probed the possibility of increasing the yield of the reaction by optimizing the different reaction parameters. Keeping DMF as the solvent we first screened a series of weak bases and $\mathrm{K}_{2} \mathrm{CO}_{3}$ gave the most satisfactory yields. We then hoped that with a stronger base it would be possible to reduce the amount of higher order substituted products present in the crude reaction mixture and therefore force the production of mono-functionalized material, whilst also easing the purification workload. Examples of this have been seen in the literature with respect to alkyl diol compounds, which would normally give a statistical mixture of isomers when attempting to obtain the mono-functionalized product. ${ }^{[13]}$ The dianionic species formed when $\mathrm{NaH}$ is used as the base showed a statistical bias towards the functionalization of only one hydroxyl group of $c a$. $90 \%$. A number of bases were screened, with $\mathrm{K}_{2} \mathrm{CO}_{3}$ and $\mathrm{NaH}$ giving the best results for the reaction of 2 and propargyl bromide (Table 1). Tests of different solvents indicated that DMF greatly outperformed other candidates and, although it can prove difficult to remove in a post-reaction work up, its superior yields far outweighed this drawback. The final factors to optimize were reaction time and temperature. Test reactions were run with both optimized bases with propargyl bromide over a number of different time-frames with $2 \mathrm{~h}$ best for mono-functionalized $m$ THPP. TLC analysis proved extremely reliable as an analytic tool, as each reaction consistently gave a predictable separation pattern.

Table 1. Optimization of the mono-functionalization of $\mathbf{2}$ with propargyl bromide.

\begin{tabular}{|c|c|c|c|c|c|c|}
\hline Entry & Base & Eq. & Solvent & Time & Temp. & Yield $3(\%)$ \\
\hline 1 & $\mathrm{~K}_{2} \mathrm{CO}_{3}$ & 5 & $\overline{\mathrm{DMF}}$ & $5 \mathrm{~h}$ & r.t & 18 \\
\hline 2 & $\mathrm{~K}_{3} \mathrm{PO}_{4}$ & 5 & DMF & $5 \mathrm{~h}$ & r.t & 14 \\
\hline 3 & TEA & 5 & DMF & $5 \mathrm{~h}$ & r.t & 15 \\
\hline 4 & $\mathrm{~K}_{2} \mathrm{CO}_{3}$ & 5 & DCM & $5 \mathrm{~h}$ & r.t & - \\
\hline 5 & $\mathrm{~K}_{2} \mathrm{CO}_{3}$ & 5 & Dioxane & $5 \mathrm{~h}$ & r.t & - \\
\hline 6 & $\mathrm{~K}_{2} \mathrm{CO}_{3}$ & 5 & EtOAc & $5 \mathrm{~h}$ & r.t & 2 \\
\hline 7 & $\mathrm{~K}_{2} \mathrm{CO}_{3}$ & 4 & DMF & $3 \mathrm{~h}$ & r.t & 35 \\
\hline 8 & $\mathrm{~K}_{2} \mathrm{CO}_{3}$ & 2 & DMF & $2 \mathrm{~h}$ & r.t & 48 \\
\hline 9 & $\mathrm{~K}_{2} \mathrm{CO}_{3}$ & 0.5 & DMF & $2 \mathrm{~h}$ & $50^{\circ} \mathrm{C}$ & 15 \\
\hline 10 & $\mathrm{NaH}$ & 1 & DMF & $2 \mathrm{~h}$ & r.t & 45 \\
\hline 11 & $\mathrm{NaOH}$ & 1 & DMF & $3 \mathrm{~h}$ & r.t & 23 \\
\hline 12 & Superbase ${ }^{[27]}$ & 1 & DMF & $2 \mathrm{~h}$ & r.t & 15 \\
\hline
\end{tabular}

The best conditions for the optimization of mono-functionalized $m$ THPP with propargyl bromide were found to be: 1 eq. of $\mathrm{NaH}$ as base with 2 eq. of propargyl bromide in DMF at r.t for $2 \mathrm{~h}$, resulting in $45 \%$ yield and $30 \%$ recovered starting material. Although sodium hydride gave slightly lower yields when used as a base in comparison to $\mathrm{K}_{2} \mathrm{CO}_{3}$, it was selected as the preferred option as it produced minimal quantities of the higher substituted compounds.

Higher substituted compounds were available through longer reaction times and greater equivalents of base, i.e. $\mathrm{K}_{2} \mathrm{CO}_{3}$ and propargyl bromide. The tetrasubstituted product $\mathbf{5}$ was synthesized in near quantitative yield, with the di- and trisubstituted species available in yields similar to the mono-functionalized derivative; however, these have not been optimized. These results indicated that one is able to maximize the output of mono, di, tri and tetra substituted $m$ THPP by varying the reaction time, equivalents and base used. ${ }^{[27]}$ This indicates the potential for a pathway to complicated unsymmetrical porphyrin systems in high yields, on 
both small and large scales with very cheap and accessible starting materials.

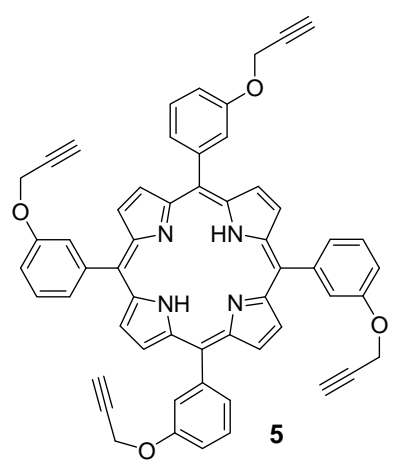

Substrate scope: To expand the scope of this synthetic methodology, a library of reactants were selected which were amenable to nucleophilic substitution chemistry, but also contained a chemically distinct functional group for use in subsequent functionalization reactions. A clear starting point for the investigation of the robustness of this method was the installation of simple aryl or alkyl chains onto the parent $m$ THPP. Starting with the conditions used in entry 8 (Table 1 ) test reactions were carried out using 1-iodopropane and benzyl chloride. Again, these reactions proved successful in obtaining mono-functionalized porphyrin products $6 \mathbf{a}$ and $7 \mathbf{a}$ in $35-40 \%$ yield and again, by altering the reaction time and equivalents, the higher order substituted products could be obtained in good yields, e.g., the tetrasubstituted product in quantitative yields (Table 2).
The next step was to test the reactivity of compounds with other substituents present on the aryl ring, which, once installed, could undergo subsequent metal-catalyzed reactions. 4-Bromobenzyl bromide and 4-benzylnitrobenzene were chosen as suitable reagents to test the effect peripheral substituents had on the reaction rate and yield. The monosubstituted derivatives $\mathbf{8 a}$ and $\mathbf{9 a}$ were isolated in $43 \%$ and $39 \%$, respectively, indicating a high tolerance for different electronic substituents on the aryl ring. Next was a probe for controlled esterification of the peripheral $\mathrm{OH}$ groups. To investigate this, 2-bromobenzoic acid and benzoic anhydride and various reaction conditions were used, however, most failed to produce significant amounts of functionalized $m$ THPP. Ultimately, 1-ethyl3-(3-dimethylaminopropyl)carbodiimide (EDC) and 1hydroxybenzotriazole $(\mathrm{HOBt})$ produced 10a and 11a in yields of $33 \%$ and $37 \%$, respectively. Again, the tetra-functionalized derivatives $\mathbf{1 0 b}$ and $\mathbf{1 1 b}$ could be obtained in near quantitative yield. This opens the possibility to conjugate a myriad of biologically interesting moieties such as sugars, bile acids and beta-lactams, as the carboxylic acid functional group is present in many biological molecules, to a symmetric porphyrin starting material, through a simple esterification reaction.

Next, we wished to expand this methodology to more modern chemistry, such as metal-catalyzed cross coupling reactions. Two of the more appealing approaches were the direct metal-catalyzed coupling of the heteroatom to a substrate, or the transformation of an $\mathrm{OH}$ into a group susceptible to classic palladium catalyzed reactions such as the Suzuki-Miyaura cross coupling.

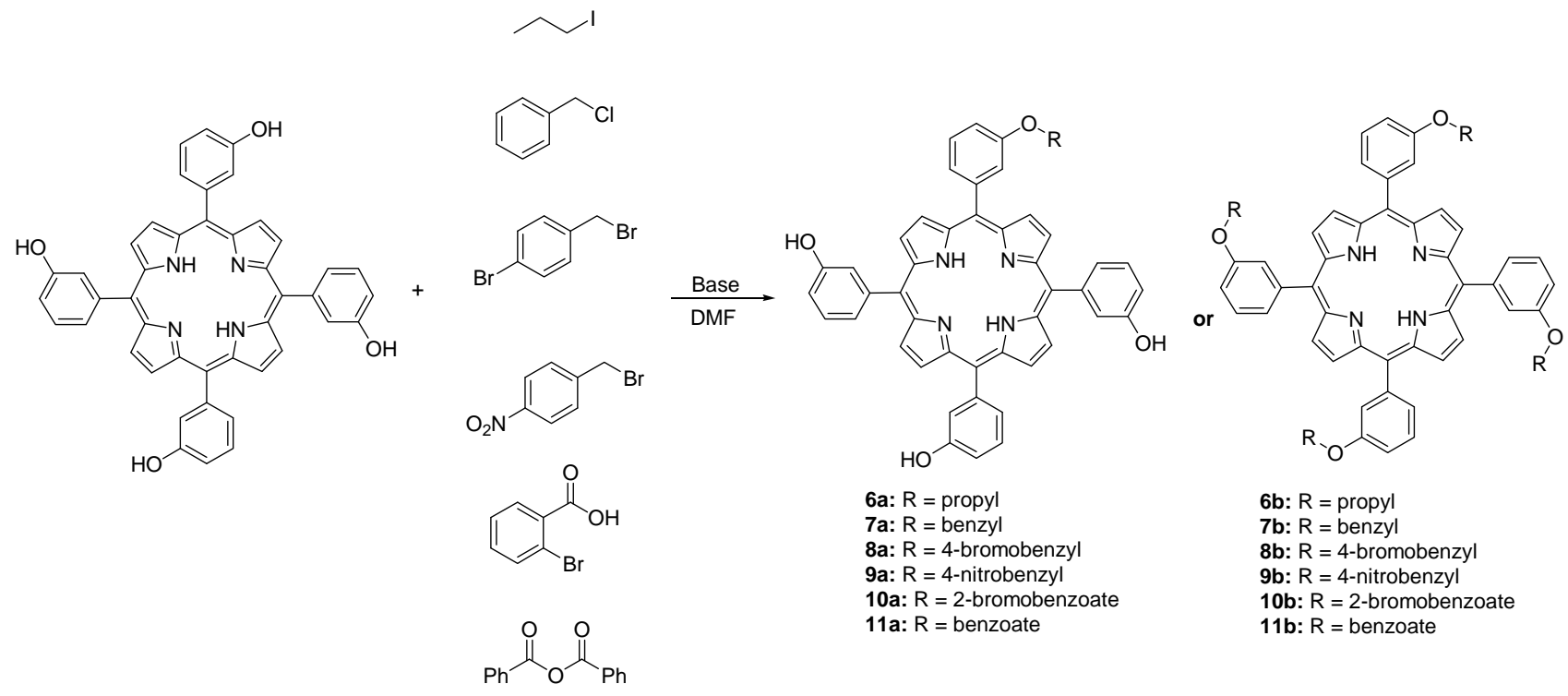

Table 2. Mono-functionalization of $m$ THPP (2) with a variety of different substrates.

\begin{tabular}{cccccccccc}
\hline Product & Reagent & Base & Eq. & Time (h) & \% Yield Mono (a) & Base & Eq. & Time (h) & \% Yield Tetra (b) \\
\hline $\mathbf{6}$ & 1-iodopropane & $\mathrm{NaH}$ & 1 & 2 & 35 & $\mathrm{~K}_{2} \mathrm{CO}_{3}$ & 10 & 13 & 95 \\
$\mathbf{7}$ & 4-benzylchloride & $\mathrm{NaH}$ & 1 & 2.5 & 44 & $\mathrm{~K}_{2} \mathrm{CO}_{3}$ & 10 & 13 & 95 \\
$\mathbf{8}$ & 4-bromobenzylbromide & $\mathrm{NaH}$ & 1 & 2 & 43 & $\mathrm{~K}_{2} \mathrm{CO}_{3}$ & 12 & 13 & 96 \\
$\mathbf{9}$ & 4-nitrobenzylbromide & $\mathrm{NaH}$ & 1 & 3 & 35 & $\mathrm{~K}_{2} \mathrm{CO}_{3}$ & 10 & 15 & 93 \\
$\mathbf{1 0}$ & 2-bromobenzoic acid & $\mathrm{NaH}$ & 1 & 3 & 40 & $\mathrm{~K}_{2} \mathrm{CO}_{3}$ & 12 & 18 & 59 \\
$\mathbf{1 1}$ & benzoic anhydride & $\mathrm{NaH}$ & 1 & 1 & 37 & $\mathrm{~K}_{2} \mathrm{CO}_{3}$ & 12 & 5 & 92 \\
\hline
\end{tabular}

Triflate and tosylate derivatives: Recent and classical publications have shown the capability of triflate and tosylate groups to react in metal-catalyzed cross-coupling reactions under a variety of different conditions and with a number of different catalyst systems. ${ }^{[14]}$ The most widely known of these is the use of the triflate group in Suzuki-Miyaura couplings. To this end, we tried to use the conditions optimized previously and synthesize a range of "activated" $m$ THPP derivatives which could be selectively functionalized in a controlled manner in a similar fashion to the nucleophilic substitution reactions.

However, reactions carried out using the optimized conditions of $\mathrm{K}_{2} \mathrm{CO}_{3}$ produced very low yields with both reagents. Yet, upon change of the base to pyridine, both trifluoromethanesulfonyl 
chloride and 4-toluenesulfonyl chloride gave the porphyrins 12a and 13a in $37 \%$ and $35 \%$ yield, respectively. Again, by increasing the equivalents of reagent and based used, the tetra-functionalized derivatives can be obtained in excellent yields (12b 92\%, 13b 90\%, Scheme 2).

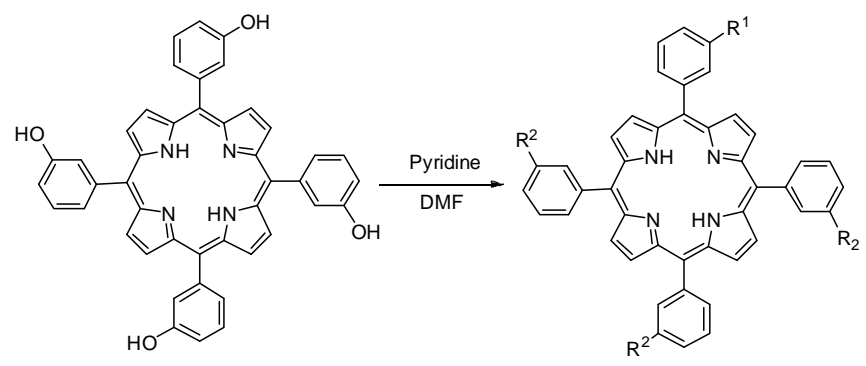

12a $R^{1}=$ triflate, $R^{2}=\mathrm{OH}(37 \%)$ 12b $R^{1}=R^{2}=$ triflate $(92 \%)$ 13a $R^{1}=$ tosylate, $R^{2}=\mathrm{OH}(35 \%)$ 13b $\mathrm{R}^{1}=\mathrm{R}^{2}=$ tosylate $(90 \%)$

Chan-Lam coupling: A number of reactions have been developed which involve an hydroxyl functionality reacting in the presence of a metal with another specific substrate. Examples are the BuchwaldHartwig reaction. ${ }^{[15]}$, the Ullmann reaction ${ }^{[16]}$ and the Chan-Lam reaction. ${ }^{[17]}$ After some initial tests, the Chan-Lam reaction showed the most promising results in terms of yield, reaction conditions, and purification. The reaction involves use of copper(II)acetate, a boronic acid and an hydroxyl or amine functionality. It is a versatile reaction, as it can proceed in a number of different solvents, at room temperature, and in an open reaction vessel. The reaction uses a stoichiometric amount of copper, hence metalloporphyrins were used to avoid copper insertion. By using a metal-catalyzed reaction, we hoped that we could achieve greater selectivity for the monosubstituted porphyrin with the higher order substitution products appearing as only minor side products.

Table 3. Results of the Chan-Lam coupling reactions of 2 with boronic acids.

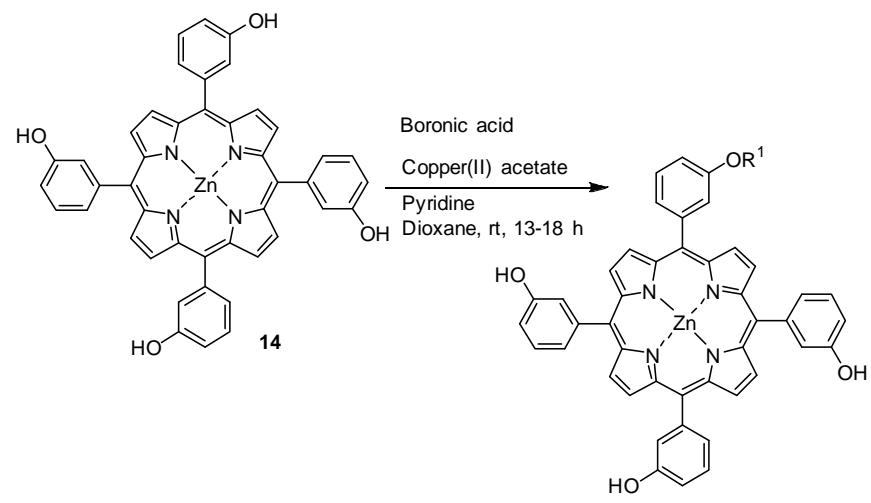

\begin{tabular}{ccccc}
\hline Product & Boronic acid & Eq. & Time (h) & \% Yield Mono \\
\hline $\mathbf{1 5}$ & phenylboronic acid & 1 & 13 & 39 \\
$\mathbf{1 6}$ & 3-cyanophenylboronic acid & 1 & 18 & 35 \\
$\mathbf{1 7}$ & 3-methoxyphenylboronic acid & 2 & 13 & 37 \\
\hline
\end{tabular}

First, the zinc(II) porphyrin $\mathbf{1 4}^{[12 \mathrm{~b}, 18]}$ was reacted with phenylboronic acid in dioxane at room temperature. After stirring for $18 \mathrm{~h}$, TLC showed the formation of $\mathbf{1 5}$ as the major product (39\%) and two faint, closely running spots, which turned out to be the two disubstituted species. ${ }^{[19]}$ After this first example of a ChanLam coupling being applied to a porphyrin system, 4cyanophenylboronic acid and 2-iodophenylboronic acid, as examples for systems amenable for post-functionalization reactions, were trialed as well. These reactions proceeded without any difficulties and the mono-functionalized species $\mathbf{1 6}$ and $\mathbf{1 7}$ were synthesized in $35 \%$ and $38 \%$ yield, respectively (Table 3 ). All three boronic acids produced satisfactory yields. As per the literature, this reaction seems to be quite tolerant of a variety of substrates under mild reaction conditions.

This provides another synthetic pathway to unsymmetric porphyrins through the one basic, readily available symmetric porphyrin. Boronic acids are easily installed into compounds through standard synthetic methodology and therefore present the potential for a large variety of possible molecules to be conjugated to these porphyrins. However, while the reactions above expands the synthetic toolkit for porphyrins a demonstration of their practical utility for the synthesis of unsymmetrical porphyrins or more complicated systems might be warranted. Somewhat arbitrarily, we chose picket-fence porphyrins and cofacial bisporphyrins as case materials.

Case study 1 - picket fence porphyrin: Picket fence porphyrins are a classic example for the impact of biomimetic model compounds. They were in vogue for many years ever since their synthesis by Collman et al. in the 1970ties ${ }^{[20]}$ as they provided researchers the first synthetic model with which to investigate the binding of $\mathrm{O}_{2}$ in heme proteins. Large libraries of these picket fenced porphyrins were synthesized, however, depending on the route selected, they all required a large number of synthetic steps, challenging chromatography and suffer from low yields.

We began with the synthesis of simple tetrasubstituted porphyrins as simple examples of picket fence porphyrins. For example, 2 was reacted with 4-tert-butylbenzylbromide or 1iodohexane using the optimized conditions to give porphyrins $\mathbf{1 8}$ and 19 (Scheme 3). These reactions worked in quantitative yield however; systems like these can also be made easily through condensation methodology. As a more 'complicated' example we targeted related $\mathrm{A}_{3} \mathrm{~B}$-type porphyrins. By reducing the equivalents and reaction time used, we were able to synthesize the trisubstituted species from both 4-tert-butylbenzyl bromide and 1-iodohexane in yields of $40 \%$ and $37 \%$, respectively.

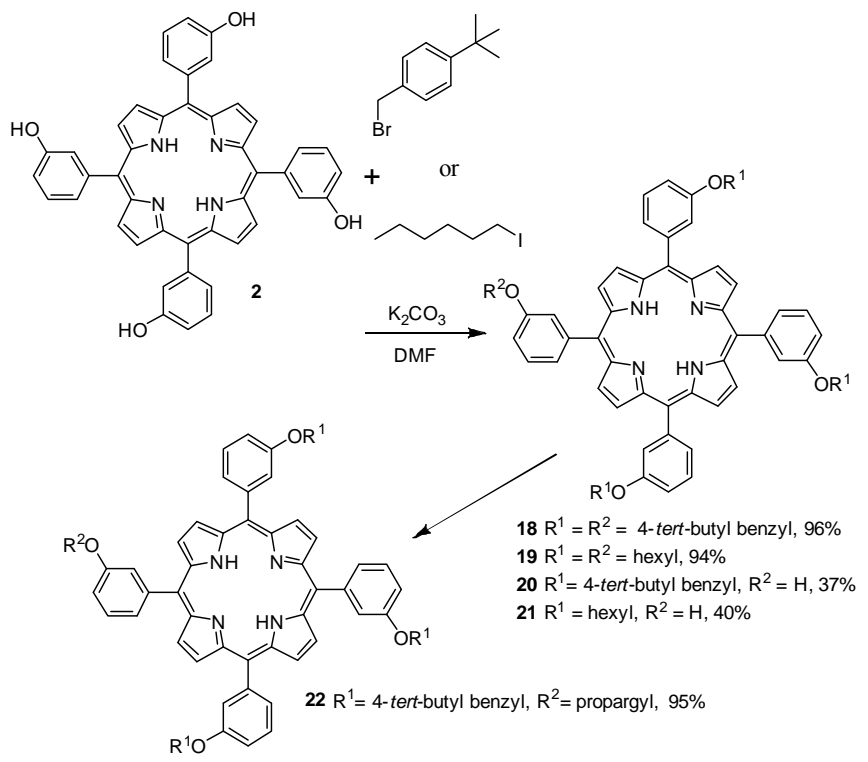

Scheme 3. Synthesis of picket-fence porphyrins through new optimized procedure. 
Once the trisubstituted species $\mathbf{2 0}$ was isolated from the reaction mixture, the final free $\mathrm{OH}$ groups were subjected to a reaction with propargyl bromide. This reaction proceeded in high yield and produced a stable "picket fence porphyrin mimic" 22, i.e., an $\mathrm{A}_{3} \mathrm{~B}$ porphyrin, with two different 'fence types'. Thus, by taking 2 as the starting material, a synthetically difficult class of porphyrin can be produced in two steps using simple and cost effective chemistry in far greater yields compared to the alternative mixed condensation or total synthesis route. By applying this methodology to $o$-THPP ${ }^{[21]}$, once synthesized, these compounds could potentially undergo atropisomer resolution to afford the $\alpha, \alpha, \alpha, \alpha$-derivative, i.e., a true picket fence porphyrin.

Case study 2 - cofacial bisporphyrins: Another example of porphyrins systems frequently used in model studies is porphyrin arrays and notably cofacial bisporphyrins. These serve as metalloenzyme $\mathrm{e}^{[4 \mathrm{a}, 22]}$ or special pair mimics in photosynthesis ${ }^{[23]}$ and are suitable models for multi-electron redox reactions. Many of these require lengthy syntheses, can typically contain unstable amide linker groups and suffer from low yields. We wished to extend the methodology developed previously and apply it to the synthesis of cofacial porphyrin cages. ${ }^{[2]}$ By using alkyl chains, it was hoped that a degree of flexibility could be imparted into the cage system so as to enable the molecule to adopt the optimum metal-metal distance needed for catalytic activity or substrate binding. The synthesis proceeded again with 2 as the starting material and reacting it with an excess of 1,4-dibromobutane using $\mathrm{K}_{2} \mathrm{CO}_{3}$ as a base, affording the tetrasubstituted compound $\mathbf{2 4}$ in near quantitative yield. The resultant primary alkyl bromides can react again in a simple nucleophilic substitution reaction with one equivalent of $(5,10,15,20$-tetrakis(3-hydroxyphenyl)porphyrinato)cobalt(II) [Co(II) $m$ THPP, 24] to afford a cofacial bisporphyrin 25 with a free base and $\mathrm{Co}(\mathrm{II})$ porphyrin unit in a yield of $12 \%$ (Scheme 4). ${ }^{[25]}$

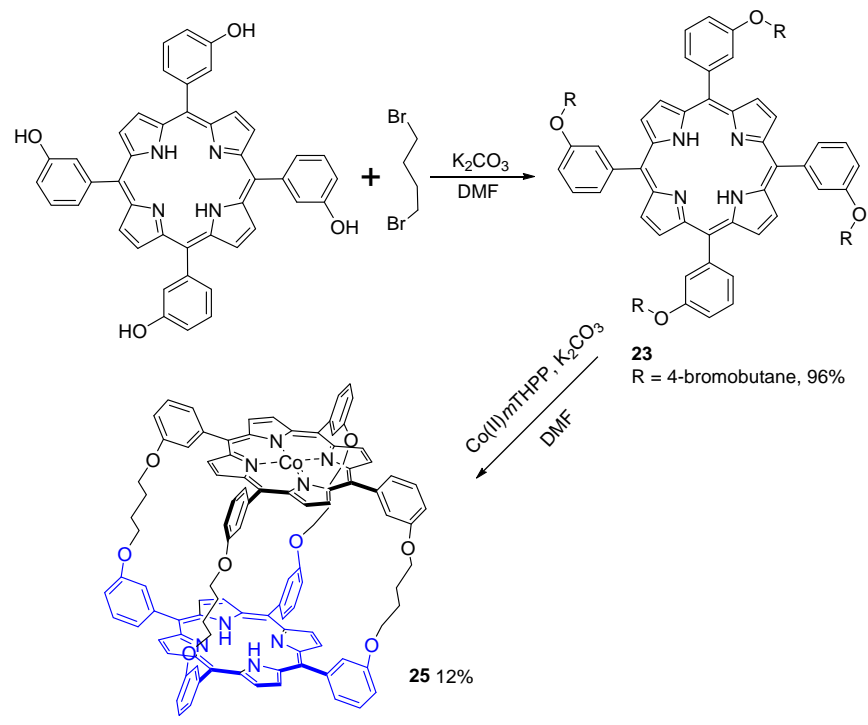

Scheme 4. Synthesis of the cofacial bisporphyrin cage 24 .

The use of a cobalt porphyrin for the synthesis of the cage was to exemplify the ease at which one could make potential four electron catalysts. This simple synthesis can be modified to incorporate linkers of different length and type, along with the ability to include different metal systems. With the wide range of versatility possible from this methodology, other applications, e.g. the synthesis of artificial photosynthetic reaction centers are possible. ${ }^{[5 \mathrm{~d}, 23 \mathrm{a}]}$ The obvious strength of this synthetic pathway is that one can choose almost any metal center for the porphyrin core along with the option of having mixed metal centers or even co-facial free base versus metal porphyrins along with a variety of different linkers, all of which are examples of reduced symmetry cage systems. These historically difficult compounds can now be made in two steps, again by using simple, cheap and reliable chemistry.

\section{Conclusion}

A library of monosubstituted $m$ THPP derivatives could be successfully be synthesized through a number of simple reactions pathways. Using historic phenolic chemistry, compounds were synthesized using $\mathrm{S}_{\mathrm{N}}$, esterification and $\mathrm{OH}$ activation reactions. This chemistry was updated for porphyrins by using the recently discovered Chan-Lam copper-catalyzed coupling of a heteroatom with a boronic acid. This allowed the synthesis of some simple unsymmetrical porphyrin systems as a demonstration for the ease with which one can quickly generate historically cumbersome compounds, e.g., picket-fence and cofacial porphyrins, in few synthetic steps using the new methodology. Whilst this is certainly not a conditio sine qua non for the optimum preparation of unsymmetrical porphyrins, it presents a rapid, robust and above all cost-effective strategy for the synthesis of a variety of soluble, unsymmetrical porphyrins that can be fine-tuned for a plethora of applications, all starting from one symmetric parent porphyrin molecule.

\section{Experimental Section}

General methods: Instrumentation and standard techniques used were as described elsewhere. ${ }^{[26]}$ Microwave reactions were carried out on a Sharp El-254v microwave oven.

General Procedure A - Microwave assisted condensation of mTHPP 2: ${ }^{[18]}$ Propionic acid $(3.5 \mathrm{~mL})$ and nitrobenzene $(1.5 \mathrm{~mL})$ were added to a Pyrex Erlenmeyer flask (100 $\mathrm{mL})$. Pyrrole $(0.74 \mathrm{~mL})$ and 3-hydroxybenzaldehyde $(1 \mathrm{~mL})$ were added and the reaction vessel was sealed with hydrophilic cotton wool in order to prevent spillages and, most importantly, to avoid pressure build-ups associated with hazardous explosion risks. The yellowish reaction mixture was placed at the center of the rotating plate in the microwave oven and heated for $5 \mathrm{~min}(1 \mathrm{~min}$ periods intercalated by $3 \mathrm{~min}$ intervals with the power off to avoid overheating) at $640 \mathrm{~W}$. The reaction was monitored over time by UV-vis absorption spectroscopy. The cooled crude reaction mixture was washed with $\mathrm{MeOH}$ and filtered. The liquid extract was evaporated under reduced pressure and the resulting residue was taken into ethyl acetate. Purification was achieved through flash chromatography packed with silica gel and eluted with ethyl acetate $/ \mathrm{CH}_{2} \mathrm{Cl}_{2}=1: 1(\mathrm{v} / \mathrm{v})$. The red fraction was collected and recrystallized from ethyl acetate $/ n$-hexane, yielding 2 in $22 \%$ yield.

General procedure B - Mono-functionalization of $\boldsymbol{m}$ THPP: $m$ THPP (1 eq.) was added to a round bottomed flask $(100 \mathrm{~mL})$ and charged with DMF $(10 \mathrm{~mL})$ and $\mathrm{NaH}(1$ eq.). The reaction was left to stir for 30 minutes under argon before the appropriate functionalization reagent (2-3 eq.) was added. The reaction mixture was monitored by TLC and once full conversion was observed the reaction was terminated through the addition of $\mathrm{CH}_{2} \mathrm{Cl}_{2}(50 \mathrm{~mL})$. The crude reaction mixture was washed with distilled water $(1 \times 30 \mathrm{~mL})$, sat. aq. $\mathrm{NaHCO}_{3}(1 \times 30 \mathrm{~mL})$, brine $(1 \times 30 \mathrm{~mL})$, and distilled water $(2 \times 30 \mathrm{~mL})$. The organic phase was dried over anhydrous sodium sulfate, filtered and evaporated under reduced pressure. Crude products were typically purified by column chromatography on silica gel using $\mathrm{CH}_{2} \mathrm{Cl}_{2} / n$-hexane/MeOH (3:1:0.2).

General Procedure C - Di-, tri- and tetra-functionalization of $\boldsymbol{m T H P P}: m$ THPP (1 eq.) was added to a round bottomed flask $(100 \mathrm{~mL})$ and charged with DMF $(10 \mathrm{~mL})$ and $\mathrm{K}_{2} \mathrm{CO}_{3}$ (5-20 eq.). The reaction was left to stir for 30 minutes under argon before the appropriate functionalization reagent (10-20 eq.) was added. The reaction mixture was monitored by TLC and once full conversion to the desired degree of substitution was observed the reaction was terminated through the addition of $\mathrm{CH}_{2} \mathrm{Cl}_{2}(50 \mathrm{~mL})$. The work-up proceeded as per general procedure B.

General Procedure D - Chan-Lam couplings of $\boldsymbol{m}$ THPP: $m$ THPP (1 eq.) was added to a round bottomed flask $(100 \mathrm{~mL})$ and charged with dioxane $(10 \mathrm{~mL}), \mathrm{Cu}(\mathrm{II}) \mathrm{OAc}_{2}(2$ eq.), boronic acid ( 2 eq.) and pyridine $(0.2 \mathrm{~mL})$. The reaction mixture was monitored by TLC and once full conversion to the desired degree of substitution was observed the reaction was terminated through the addition of $\mathrm{CH}_{2} \mathrm{Cl}_{2}(50 \mathrm{~mL})$. The work-up proceeded as per general procedure $\mathrm{B}$. 
5,10,15,20-Tetrakis[3-(prop-2-yn-1-yloxy)phenyl]porphyrin 5: The compound was synthesized using General Procedure $\mathrm{C}$ : a $50 \mathrm{~mL}$ round bottom flask containing $10 \mathrm{~mL}$ of DMF, $2(400 \mathrm{mg}, 0.59 \mathrm{mmol}), \mathrm{K}_{2} \mathrm{CO}_{3}(815 \mathrm{mg}, 5.9 \mathrm{mmol})$ and propargyl bromide $(0.45 \mathrm{~mL}, 5.9 \mathrm{mmol})$ was allowed stir for $5 \mathrm{~h}$. Yield: $392 \mathrm{mg}$ of purple crystals $(0.472$ mmol, 98\%). m.p. $>300{ }^{\circ} \mathrm{C} ; R_{f}=0.88\left(\mathrm{CH}_{2} \mathrm{Cl}_{2} / n\right.$-hexane/MeOH $\left.=3: 1: 0.2, \mathrm{v} / \mathrm{v} / \mathrm{v}\right) ;{ }^{1} \mathrm{H}$ NMR $\left(400 \mathrm{MHz}, \mathrm{CDCl}_{3}, 25^{\circ} \mathrm{C}\right): \delta=-2.82(\mathrm{~s}, \mathrm{br}, 2 \mathrm{H}, \mathrm{NH}), 2.57(\mathrm{~s}, 4 \mathrm{H}$, alkyne- $H$ ), 4.87 $\left(\mathrm{s}, 8 \mathrm{H},-\mathrm{CH}_{2}, 7.40\left(\mathrm{~d},{ }^{3} J_{\mathrm{H}-\mathrm{H}}=7.81 \mathrm{~Hz}, 4 \mathrm{H}, \mathrm{Ar}-\mathrm{H}\right), 7.65\left(\mathrm{t},{ }^{3} J_{\mathrm{H}-\mathrm{H}}=7.81 \mathrm{~Hz}, 4 \mathrm{H}, m-\mathrm{Ph}\right)\right.$, $7.85(\mathrm{~m}, 8 \mathrm{H}, A r-H), 8.88 \mathrm{ppm}\left(\mathrm{s}, 8 \mathrm{H}, H_{\beta}\right) ;{ }^{13} \mathrm{C}$ NMR $\left(100 \mathrm{MHz}, \mathrm{CDCl}_{3}, 25{ }^{\circ} \mathrm{C}\right): \delta=$ 56.1 $75.9,78.6,114.6,119.6,119.7,121.4,125.6,127.6,128.6,133.8,143.5,143.51$ $155.9,157.2 \mathrm{ppm}$; UV/Vis (EtOAc): $\lambda_{\max }(\lg \varepsilon)=419$ (5.47), 512 (4.03), $546(3.04), 590$ (2.10), $646 \mathrm{~nm}$ (1.35); HRMS (MALDI) calcd for [ $\left.\mathrm{C}_{56} \mathrm{H}_{38} \mathrm{~N}_{4} \mathrm{O}_{4}\right][\mathrm{M}]+830.2893$, found 830.2896 .

5,10,15-Tris(3-hydroxyphenyl)-20-[(propoxy)phenyl]porphyrin 6a: Synthesized via General Procedure B using 2 (400 mg, $0.59 \mathrm{mmol})$, NaH $(14.2 \mathrm{mg}, 0.59 \mathrm{mmol})$, and 1 iodopropane $(0.12 \mathrm{~mL}, 1.2 \mathrm{mmol})$. The reaction was complete after $2 \mathrm{~h}$. Yield: $149 \mathrm{mg}$ of purple crystals $(0.32 \mathrm{mmol}, 35 \%)$. M.p. $>300{ }^{\circ} \mathrm{C} ; R_{f}=0.38\left(\mathrm{CH}_{2} \mathrm{Cl}_{2} / n\right.$-hexane $/ \mathrm{MeOH}$ $=3: 1: 0.2, \mathrm{v} / \mathrm{v} / \mathrm{v}) ;{ }^{1} \mathrm{H}$ NMR $\left(400 \mathrm{MHz},\left(\mathrm{CDCl}_{3}\right), 25^{\circ} \mathrm{C}\right): \delta_{\mathrm{H}}=-2.88(\mathrm{~s}, \mathrm{br}, 2 \mathrm{H}, \mathrm{N} H), 0.87$ $\left(\mathrm{t},{ }^{3} \mathrm{~J}_{\mathrm{H}-\mathrm{H}}=7.27 \mathrm{~Hz}, 3 \mathrm{H},-\mathrm{CH}_{3}\right), 1.87,\left(\mathrm{q},{ }^{3} J_{\mathrm{H}-\mathrm{H}}=7.27 \mathrm{~Hz}, 2 \mathrm{H},-\mathrm{CH}_{2}\right), 4.08\left(\mathrm{t},{ }^{3} J_{\mathrm{H}-\mathrm{H}}=7.27\right.$ $\left.\mathrm{Hz}, 2 \mathrm{H}, \mathrm{O}-\mathrm{CH}_{2}\right), 7.17\left(\mathrm{t},{ }^{3} J_{\mathrm{H}-\mathrm{H}}=6.65 \mathrm{~Hz}, 3 \mathrm{H}, A r-\mathrm{H}\right), 7.29\left(\mathrm{~d},{ }^{3} J_{\mathrm{H}-\mathrm{H}}=6.65 \mathrm{~Hz}, 1 \mathrm{H}, A r\right.$ $\mathrm{H}), 7.47\left(\mathrm{t},{ }^{3} J_{\mathrm{H}-\mathrm{H}}=6.65 \mathrm{~Hz}, 3 \mathrm{H}, A r-\mathrm{H}\right), 7.61(\mathrm{~m}, 7 \mathrm{H}, A r-\mathrm{H}), 7.75(\mathrm{~m}, 2 \mathrm{H}, A r-\mathrm{H}), 8.79$ $\left(\mathrm{m}, 2 \mathrm{H}, H_{\beta}\right) 8.85 \mathrm{ppm}\left(\mathrm{m}, 6 \mathrm{H}, H_{\beta}\right) ;{ }^{13} \mathrm{C}$ NMR $\left(100 \mathrm{MHz},\left(\mathrm{CD}_{3}\right)_{2} \mathrm{SO}_{2}\right): \delta=31.6,36.6$ 69.7, 114.8, 119.7, 122.0, 127.1, 127.5, 126.2, 127.5, 154.6, 157.4, 162.7 ppm; UV/Vi (EtOAc): $\lambda_{\max }(\lg \varepsilon)=417$ (5.02), 515 (3.76), 547 (3.50), 590 (3.13), $647 \mathrm{~nm}(2.95)$; HRMS (MALDI): $m / z$ calcd for $\left[\mathrm{C}_{47} \mathrm{H}_{36} \mathrm{~N}_{4} \mathrm{O}_{4}\right][\mathrm{M}+]$ : 720.2737 , found 720.2734 .

5,10,15,20-Tetra[(propoxy)phenyl]porphyrin 6b: Synthesized via General Procedure $\mathrm{C}$ using $2(400 \mathrm{mg}, 0.59 \mathrm{mmol}), \mathrm{K}_{2} \mathrm{CO}_{3}(815 \mathrm{mg}, 5.9 \mathrm{mmol})$, and 1-iodopropane (0.6 $\mathrm{mL}, 5.9 \mathrm{mmol})$. The reaction was complete after $13 \mathrm{~h}$. Yield: $484 \mathrm{mg}$ of purple crystals $(0.57 \mathrm{mmol}, 95 \%)$. m.p. $>300{ }^{\circ} \mathrm{C} ; R_{f}=0.95\left(\mathrm{CH}_{2} \mathrm{Cl}_{2} / n\right.$-hexane $\left./ \mathrm{MeOH}=3: 1: 0.2, \mathrm{v} / \mathrm{v} / \mathrm{v}\right)$ ${ }^{1} \mathrm{H}$ NMR $\left(400 \mathrm{MHz}, \mathrm{CDCl}_{3}, 25^{\circ} \mathrm{C}\right): \delta_{\mathrm{H}}=-2.77(\mathrm{~s}, 2 \mathrm{H}, \mathrm{NH}), 1.10\left(\mathrm{t},{ }^{3} J_{\mathrm{H}-\mathrm{H}}=7.4 \mathrm{~Hz}\right.$ $12 \mathrm{H}$, propane), $1.91\left(\mathrm{q},{ }^{3} J_{\mathrm{H}-\mathrm{H}}=7.4 \mathrm{~Hz}, 8 \mathrm{H}\right.$, propane), $4.13\left(\mathrm{t},{ }^{3} J_{\mathrm{H}-\mathrm{H}}=6.5 \mathrm{~Hz}, 8 \mathrm{H}, \mathrm{O}-\mathrm{CH} \mathrm{H}_{2}\right.$, $7.35(\mathrm{~m}, 4 \mathrm{H}, \operatorname{Ar}-H), 7.64\left(\mathrm{t},{ }^{3} J_{\mathrm{H}-\mathrm{H}}=7.5 \mathrm{~Hz}, 4 \mathrm{H}, m-\mathrm{Ph}\right), 7.80(\mathrm{~m}, 8 \mathrm{H}, \mathrm{Ar}-H), 8.92 \mathrm{ppm}$ $\left(\mathrm{m}, 8 \mathrm{H}, H_{\beta}\right) ;{ }^{13} \mathrm{C}$ NMR $\left(100 \mathrm{MHz}, \mathrm{CDCl}_{3}, 25{ }^{\circ} \mathrm{C}\right): \delta=22.7,29.7,69.7,114.2,119.9$ $121.0,127.4,127.5,143.4,157.5 \mathrm{ppm}$; UV/Vis (EtOAc): $\lambda_{\max }(\lg \varepsilon)=418(5.04), 515$ (3.75), 547 (3.53), 590 (3.13), $647 \mathrm{~nm}$ (2.99); HRMS (MALDI) $\mathrm{m} / z$ calcd for $\left[\mathrm{C}_{56} \mathrm{H}_{54} \mathrm{~N}_{4} \mathrm{O}_{4}\right]\left[\mathrm{M}^{+}\right]: 846.4145$, found 846.4141 .

5-[(4-benzyloxy)10,15,20-tris(3-hydroxyphenyl)]porphyrin 7a: Synthesized via General Procedure B using $2(400 \mathrm{mg}, 0.59 \mathrm{mmol}), \mathrm{NaH}(14.2 \mathrm{mg}, 0.59 \mathrm{mmol})$, and benzyl chloride $(0.067 \mathrm{~mL}, 0.59 \mathrm{mmol})$. The reaction was complete after $2.5 \mathrm{~h}$. Yield $199 \mathrm{mg}$ of purple crystals $(0.23 \mathrm{mmol}, 44 \%)$. m.p. $>300{ }^{\circ} \mathrm{C} ; R_{f}=0.38\left(\mathrm{CH}_{2} \mathrm{Cl}_{2} / n\right.$ hexane $/ \mathrm{MeOH}=3: 1: 0.2, \mathrm{v} / \mathrm{v} / \mathrm{v}) ;{ }^{1} \mathrm{H}$ NMR $\left(400 \mathrm{MHz},\left(\mathrm{CD}_{3}\right)_{2} \mathrm{SO}, 25{ }^{\circ} \mathrm{C}\right): \delta_{\mathrm{H}}=-2.99(\mathrm{~s}$ $2 \mathrm{H}, \mathrm{NH}), 5.29\left(\mathrm{~s}, 2 \mathrm{H},-\mathrm{CH}_{2}\right), 7.23\left(\mathrm{~d},{ }^{3} J_{\mathrm{H}-\mathrm{H}}=7.41 \mathrm{~Hz}, 3 \mathrm{H}, \mathrm{Ar}-H\right), 7.33\left(\mathrm{~d},{ }^{3} J_{\mathrm{H}-\mathrm{H}}=7.41\right.$ $\mathrm{Hz}, 1 \mathrm{H}, \operatorname{Ar}-H), 7.39\left(\mathrm{t},{ }^{3} J_{\mathrm{H}-\mathrm{H}}=7.41 \mathrm{~Hz}, 3 \mathrm{H}, \operatorname{Ar}-H\right), 7.52\left(\mathrm{~d},{ }^{3} J_{\mathrm{H}-\mathrm{H}}=7.41 \mathrm{~Hz}, 3 \mathrm{H}, \operatorname{Ar}-H\right)$ $7.59(\mathrm{~m}, 9 \mathrm{H}, \mathrm{Ar}-H), 7.69(\mathrm{~m}, 1 \mathrm{H}, \mathrm{Ar}-H), 7.87(\mathrm{~s}, 1 \mathrm{H}, \mathrm{Ar}-H), 8.82\left(\mathrm{~d},{ }^{3} J_{\mathrm{H}-\mathrm{H}}=4.84 \mathrm{~Hz}\right.$ $\left.2 \mathrm{H}, H_{\beta}\right), 8.82\left(\mathrm{~d},{ }^{3} J_{\mathrm{H}-\mathrm{H}}=4.84 \mathrm{~Hz}, 6 \mathrm{H}, H_{\beta}\right), 9.88(\mathrm{~s}, 3 \mathrm{H},-\mathrm{OH}) ;{ }^{13} \mathrm{C}$ NMR $(150 \mathrm{MHz}$, $\left.\mathrm{CDCl}_{3}\right): \delta=69.9,115.5,119.9,120.4,120.4,122.3,122.4,126.2,128.3,128.9,130.9$ $137.5,142.8,142.9,156.2,157.3 \mathrm{ppm} ; \mathrm{UV} / \mathrm{Vis}(\mathrm{EtOAc}): \lambda_{\max }(\lg \varepsilon)=417(5.41), 514$ (4.08), 547 (3.69), 591 (3.59), $648 \mathrm{~nm}$ (3.48); HRMS (MALDI) $\mathrm{m} / z$ calcd for $\left[\mathrm{C}_{51} \mathrm{H}_{36} \mathrm{~N}_{4} \mathrm{O}_{4}\right][\mathrm{M}+]:$ 768.2737, found 768.2740.

5,10,15,20-Tetrakis[(3-benzyloxy)phenyl]porphyrin 7b: Synthesized via General Procedure C using 2 (400 mg, $0.59 \mathrm{mmol}), \mathrm{K}_{2} \mathrm{CO}_{3}(815 \mathrm{mg}, 5.9 \mathrm{mmol})$, and 4 benzylchloride $(0.68 \mathrm{~mL}, 5.9 \mathrm{mmol})$. The reaction was complete after $13 \mathrm{~h}$. Yield: 582 mg of purple crystals $(0.56 \mathrm{mmol}, 95 \%)$. m.p. $>300{ }^{\circ} \mathrm{C} ; R_{f}=0.95\left(\mathrm{CH}_{2} \mathrm{Cl}_{2} / n\right.$ hexane $/ \mathrm{MeOH}=3: 1: 0.2, \mathrm{v} / \mathrm{v} / \mathrm{v}) ;{ }^{1} \mathrm{H}$ NMR $\left(400 \mathrm{MHz}, \mathrm{CDCl}_{3}, 25^{\circ} \mathrm{C}\right): \delta_{\mathrm{H}}=-2.60(\mathrm{~s}, 2 \mathrm{H}$ $\mathrm{N} H), 5.32\left(\mathrm{~s}, 8 \mathrm{H},-\mathrm{CH}_{2}\right), 7.41(\mathrm{~m}, 4 \mathrm{H}, \mathrm{Ar}-H), 7.48\left(\mathrm{t},{ }^{3} J_{\mathrm{H}-\mathrm{H}}=7.07 \mathrm{~Hz}, 12 \mathrm{H}, \mathrm{Ar}-H\right), 7.59$ $\left(\mathrm{d},{ }^{3} J_{\mathrm{H}-\mathrm{H}}=7.57 \mathrm{~Hz}, 8 \mathrm{H}, \mathrm{Ar}-H\right), 7.74\left(\mathrm{t},{ }^{3} J_{\mathrm{H}-\mathrm{H}}=7.57 \mathrm{~Hz}, 4 \mathrm{H}, \mathrm{Ar}-H\right), 7.98\left(\mathrm{~d},{ }^{3} J_{\mathrm{H}-\mathrm{H}}=7.57\right.$ $\mathrm{Hz}, 4 \mathrm{H}, \mathrm{Ar}-H), 8.03(\mathrm{~s}, 4 \mathrm{H}, \mathrm{Ar}-H), 9.02 \mathrm{ppm}\left(\mathrm{m}, 8 \mathrm{H}, H_{\beta}\right) ;{ }^{13} \mathrm{C} \mathrm{NMR}\left(100 \mathrm{MHz}, \mathrm{CDCl}_{3}\right.$ $\left.25^{\circ} \mathrm{C}\right): \delta=70.3,114.6,119.9,121.6,127.6,127.7,128.1,128.6,128.7,128.8,137.0$ 143.5, $157.2 \mathrm{ppm}$; UV/Vis (EtOAc): $\lambda_{\max }(\lg \varepsilon)=417$ (5.02), 515 (3.76), 547 (3.50), 590 (3.15), $647 \mathrm{~nm}$ (2.97); HRMS (MALDI) $\mathrm{m} / z$ calcd for $\left[\mathrm{C}_{72} \mathrm{H}_{54} \mathrm{~N}_{4} \mathrm{O}_{4}\right]\left[\mathrm{M}^{+}\right]: 1038.4145$, found 1038.4140

\section{5-[3-(4-Bromobenzyloxy)phenyl]-10,15,20-tris(3-hydroxyphenyl)porphyrin}

8a:

Synthesized via General Procedure B using 2 ( $400 \mathrm{mg}, 0.59 \mathrm{mmol}), \mathrm{NaH}(14.2 \mathrm{mg}, 0.59$ $\mathrm{mmol}$ ), and $p$-bromobenzyl bromide $(292 \mathrm{mg}, 1.2 \mathrm{mmol})$. The reaction was completed after $2 \mathrm{~h}$. Yield: $215 \mathrm{mg}$ of purple crystals $(0.25 \mathrm{mmol}, 43 \%)$. m.p. $>300{ }^{\circ} \mathrm{C} ; R_{f}=0.2$ $\left(\mathrm{CH}_{2} \mathrm{Cl}_{2} / n\right.$-hexane/MeOH $\left.=3: 1: 0.2, \mathrm{v} / \mathrm{v} / \mathrm{v}\right) ;{ }^{1} \mathrm{H}$ NMR $\left(400 \mathrm{MHz},\left(\mathrm{CD}_{3}\right)_{2} \mathrm{SO}, 25{ }^{\circ} \mathrm{C}\right): \delta_{\mathrm{H}}$ $=-2.98(\mathrm{~s}, 2 \mathrm{H},-\mathrm{N} H), 5.25\left(\mathrm{~s}, 2 \mathrm{H},-\mathrm{CH}_{2}\right), 7.22\left(\mathrm{~d},{ }^{3} J_{\mathrm{H}-\mathrm{H}}=7.73 \mathrm{~Hz}, 3 \mathrm{H}, \mathrm{Ar}-H\right), 7.42(\mathrm{t}$ $\left.{ }^{3} J_{\mathrm{H}-\mathrm{H}}=7.73 \mathrm{~Hz} 3 \mathrm{H}, \mathrm{Ar}-H\right), 7.57(\mathrm{~m}, 12 \mathrm{H}, \mathrm{Ar}-H), 7.67\left(\mathrm{t},{ }^{3} J_{\mathrm{H}-\mathrm{H}}=7.73 \mathrm{~Hz}, 1 \mathrm{H}\right.$, Ar- $H$ ), $7.76(\mathrm{~m}, 1 \mathrm{H}, \mathrm{Ar}-H), 7.81(\mathrm{~m}, 1 \mathrm{H}, \mathrm{Ar}-H), 8.77\left(\mathrm{~m}, 2 \mathrm{H}, H_{\beta}\right), 8.88\left(\mathrm{~m}, 6 \mathrm{H}, H_{\beta}\right), 9.87(\mathrm{~s}$ $3 \mathrm{H},-\mathrm{OH} ;{ }^{13} \mathrm{C}$ NMR $\left(150 \mathrm{MHz}, \mathrm{CDCl}_{3}\right): 69.1,115.4,115.5,119.9,120.3,120.4,121.4$ $121.7,122.3,126.2,128.1,128.3,128.4,130.3,131.9,136.9,142.8,156.2,156.9 \mathrm{ppm}$ UV/Vis (EtOAc): $\lambda_{\max }(\lg \varepsilon)=417$ (5.40), $514(4.07), 547(3.67), 591(3.58), 648 \mathrm{~nm}$ (3.47) ; HRMS (MALDI): $\mathrm{m} / z$ calcd for $\left[\mathrm{C}_{51} \mathrm{H}_{35} \mathrm{BrN}_{4} \mathrm{O}_{4}\right]$ [M+]: 846.1842, found 846.1861 .

5,10,15,20-Tetrakis[3-(4-bromobenzyloxy)phenyl]porphyrin 8b: Synthesized via General Procedure C using 2 (400 mg, $0.59 \mathrm{mmol}), \mathrm{K}_{2} \mathrm{CO}_{3}(815 \mathrm{mg}, 5.9 \mathrm{mmol})$, and $p$ bromobenzyl bromide $(1.75 \mathrm{~g}, 7.1 \mathrm{mmol})$. The reaction was completed after $13 \mathrm{~h}$. Yield: $765 \mathrm{mg}$ purple crystals $(0.56 \mathrm{mmol}, 96 \%)$. m.p. $>300{ }^{\circ} \mathrm{C} ; R_{f}=0.91\left(\mathrm{CH}_{2} \mathrm{Cl}_{2} / n\right.$ hexane $/ \mathrm{MeOH}=3: 1: 0.2, \mathrm{v} / \mathrm{v} / \mathrm{v}) ;{ }^{1} \mathrm{H}$ NMR $\left(400 \mathrm{MHz}, \mathrm{CDCl}_{3}, 25^{\circ} \mathrm{C}\right): \delta_{\mathrm{H}}=-2.91(\mathrm{~s}, 2 \mathrm{H})$ $5.19\left(\mathrm{~s}, 8 \mathrm{H},-\mathrm{OCH}_{2}\right), 7.32\left(\mathrm{~d},{ }^{3} J_{\mathrm{H}-\mathrm{H}}=7.8 \mathrm{~Hz}, 4 \mathrm{H}, \mathrm{Ar}-\mathrm{H}\right), 7.45\left(\mathrm{~d},{ }^{3} J_{\mathrm{H}-\mathrm{H}}=8.64 \mathrm{~Hz}, 8 \mathrm{H}, \mathrm{o}-\right.$ $\mathrm{PhBr}), 7.65\left(\mathrm{t},{ }^{3} J_{\mathrm{H}-\mathrm{H}}=5.5 \mathrm{~Hz}, 4 \mathrm{H}, \mathrm{Ar}-H_{m}\right), 7.81(\mathrm{~s}, 4 \mathrm{H}, \mathrm{Ar}-H), 7.86\left(\mathrm{~d},{ }^{3} J_{\mathrm{H}-\mathrm{H}}=6.2 \mathrm{~Hz}\right.$
$4 \mathrm{H}, \mathrm{Ar}-H), 8.04\left(\mathrm{~d},{ }^{3} J_{\mathrm{H}-\mathrm{H}}=8.64 \mathrm{~Hz}, 8 \mathrm{H}, \mathrm{Ar}-H\right), 8.82 \mathrm{ppm}\left(\mathrm{d},{ }^{3} J_{\mathrm{H}-\mathrm{H}}=3.8 \mathrm{~Hz}, 8 \mathrm{H}, H_{\beta}\right)$; ${ }^{13} \mathrm{C}$ NMR $\left(150 \mathrm{MHz}, \mathrm{CDCl}_{3}\right): \delta=68.7,114.5,119.7,121.5123 .5,123.6,123.9,127.5$, $127.9,128.3,128.5,130.7,143.4,144.2,147.4,156.5 \mathrm{ppm}$; UV/Vis (EtOAc): $\lambda_{\max }(\lg \varepsilon$ $=417$ (5.02), 515 (3.76), 547 (3.50), $592(3.10), 647 \mathrm{~nm}$ (3.15); HRMS (MALDI): $m / z$ calcd for $\left[\mathrm{C}_{72} \mathrm{H}_{50} \mathrm{Br}_{4} \mathrm{~N}_{4} \mathrm{O}_{4}\right]\left[\mathrm{M}^{+}\right]$: 1350.0566 , found 1350.0520 .

5,10,15-Tris(3-hydroxyphenyl)-20-[3-(4-nitrobenzyloxy)phenyl]porphyrin 9a: Synthesized via General Procedure B using 2 ( $400 \mathrm{mg}, 0.59 \mathrm{mmol})$, NaH $(14.2 \mathrm{mg}, 0.59$ $\mathrm{mmol})$, and $p$-nitrobenzyl bromide $(254 \mathrm{mg}, 1.2 \mathrm{mmol})$. The reaction was completed after $3 \mathrm{~h}$. Yield: $187 \mathrm{mg}$ purple powder $(0.23 \mathrm{mmol}, 35 \%)$. m.p. $>300{ }^{\circ} \mathrm{C} ; R_{f}=0.23$ $\left(\mathrm{CH}_{2} \mathrm{Cl}_{2} / n\right.$-hexane/MeOH $\left.=3: 1: 0.2, \mathrm{v} / \mathrm{v} / \mathrm{v}\right) ;{ }^{1} \mathrm{H}$ NMR $\left(400 \mathrm{MHz}, \mathrm{CDCl}_{3}, 25{ }^{\circ} \mathrm{C}\right): \delta_{\mathrm{H}}=$ $2.99(\mathrm{~s}, 2 \mathrm{H}, \mathrm{N} H), 5.44\left(\mathrm{~s}, 2 \mathrm{H}, \mathrm{CH}_{2}\right), 7.20\left(\mathrm{~d},{ }^{3} J_{\mathrm{H}-\mathrm{H}}=7.54 \mathrm{~Hz}, 4 \mathrm{H}\right), 7.48(\mathrm{~m}, 1 \mathrm{H}, \mathrm{Ar}-H)$ $7.59(\mathrm{~m}, 12 \mathrm{H}, \mathrm{Ar}-H), 7.73\left(\mathrm{t},{ }^{3} J_{\mathrm{H}-\mathrm{H}}=6.43 \mathrm{~Hz}, 4 \mathrm{H}\right), 8.21\left(\mathrm{~d},{ }^{3} J_{\mathrm{H}-\mathrm{H}}=7.54 \mathrm{~Hz}, 3 \mathrm{H}, \mathrm{Ar}-H\right)$ $8.88\left(\mathrm{~m}, 8 \mathrm{H}, H_{\beta}\right) ;{ }^{13} \mathrm{C}$ NMR $\left(100 \mathrm{MHz}, \mathrm{CDCl}_{3}\right): \delta=68.7,115.5,119.8,120.4,120.5$, 121.6, 122.3 124.8, 126.2, 128.3, 128.8, 133.8, 142.8, 146.0, 147.4, 147.5, 156.2 ppm; UV/Vis (EtOAc): $\lambda_{\max }(\lg \varepsilon)=417$ (5.40), $514(3.87), 547(2.44), 591(2.91), 647 \mathrm{~nm}$ (3.13); HRMS (MALDI) $m / z$ calcd for $\left[\mathrm{C}_{51} \mathrm{H}_{35} \mathrm{~N}_{5} \mathrm{O}_{6}\right]\left[\mathrm{M}^{+}\right]$: 813.2587: found 813.2609.

5,10,15,20-Tetrakis-[3-(4-nitrobenzyloxy)phenyl]porphyrin 9b: Synthesized via General Procedure C using $2(400 \mathrm{mg}, 0.59 \mathrm{mmol}), \mathrm{K}_{2} \mathrm{CO}_{3}(815 \mathrm{mg}, 5.9 \mathrm{mmol})$, and 4 nitrobenzylbromide $(1.3 \mathrm{~g}, 5.9 \mathrm{mmol})$. The reaction was complete after $15 \mathrm{~h}$. Yield: 446 $\mathrm{mg}$ of purple powder $(0.55 \mathrm{mmol}, 93 \%)$; m.p. $>300{ }^{\circ} \mathrm{C} ; R_{f}=0.9\left(\mathrm{CH}_{2} \mathrm{Cl}_{2} / n\right.$ hexane $/ \mathrm{MeOH}=3: 1: 0.2, \mathrm{v} / \mathrm{v} / \mathrm{v}) ;{ }^{1} \mathrm{H}$ NMR $\left(400 \mathrm{MHz}, \mathrm{CDCl}_{3}, 25{ }^{\circ} \mathrm{C}\right): \delta_{\mathrm{H}}=-2.92(\mathrm{~s}, 2 \mathrm{H}$, $N \mathrm{H}), 5.19\left(\mathrm{~s}, 8 \mathrm{H},-\mathrm{OC} H_{2}\right), 7.32\left(\mathrm{~d},{ }^{3} J_{\mathrm{H}-\mathrm{H}}=7.76 \mathrm{~Hz}, 4 \mathrm{H}, \mathrm{Ar}-H\right), 7.47(\mathrm{~m}, 8 \mathrm{H}, \mathrm{Ph}-H), 7.65$ $\left(\mathrm{t},{ }^{3} J_{\mathrm{H}-\mathrm{H}}=5.48 \mathrm{~Hz}, 4 \mathrm{H}, \mathrm{Ph}-H_{m}\right), 7.81\left(\mathrm{~d},{ }^{3} J_{\mathrm{H}-\mathrm{H}}=5.48 \mathrm{~Hz}, 4 \mathrm{H}, \mathrm{Ph}-H\right), 7.86\left(\mathrm{~d},{ }^{3} J_{\mathrm{H}-\mathrm{H}}=\right.$ $6.24 \mathrm{~Hz}, 4 \mathrm{H}, \mathrm{Ph}-H), 8.04(\mathrm{~m}, 8 \mathrm{H}, \mathrm{Ph}-H), 8.82 \mathrm{ppm}\left(\mathrm{d},{ }^{3} J_{\mathrm{H}-\mathrm{H}}=3.84 \mathrm{~Hz}, 8 \mathrm{H}, H_{\beta}\right) ;{ }^{13} \mathrm{C}$ NMR $\left(150 \mathrm{MHz}, \mathrm{CDCl}_{3}\right): \delta=68.7,114.5,119.7,121.5123 .5,123.6,123.7,123.9,127.5$, $127.9,128.3,128.5,130.7,143.4,144.2,147.4,156.5 \mathrm{ppm} ; \mathrm{UV} / \mathrm{V}$ is (EtOAc): $\lambda_{\max }(\lg \varepsilon$ $=417$ (5.49), 514 (4.05), 547 (2.61), 591 (3.08), $647 \mathrm{~nm}$ (3.39); HRMS (MALDI): $\mathrm{m} / \mathrm{z}$ calcd for $\left[\mathrm{C}_{72} \mathrm{H}_{50} \mathrm{~N}_{8} \mathrm{O}_{12}\right]\left[\mathrm{M}^{+}\right]: 1218.3548$, found 1218.3539

5-[3-(2-Bromobenzoate)phenyl]-10,15,20-tris(3-hydroxyphenyl)porphyrin 10a: Synthesized via General Procedure B using 2 (400 mg, $0.59 \mathrm{mmol})$, EDC (230 mg, 1.7 $\mathrm{mmol}), N$-hydroxysuccinimide $(196 \mathrm{mg}, 1.7 \mathrm{mmol}), \mathrm{K}_{2} \mathrm{CO}_{3}(235 \mathrm{mg}, 1.7 \mathrm{mmol})$ and 2 bromobenzoic acid $(338 \mathrm{mg}, 1.7 \mathrm{mmol})$. The reaction was completed after $3 \mathrm{~h}$. Yield: $206 \mathrm{mg}$ of purple crystals $(0.24 \mathrm{mmol}, 40 \%)$; m.p. $>300{ }^{\circ} \mathrm{C} ; R_{f}=0.32\left(\mathrm{CH}_{2} \mathrm{Cl}_{2} / n-\right.$ hexane $/ \mathrm{MeOH}=3: 1: 0.2, \mathrm{v} / \mathrm{v} / \mathrm{v}) ;{ }^{1} \mathrm{H}$ NMR $\left(400 \mathrm{MHz}, \mathrm{CDCl}_{3}, 25{ }^{\circ} \mathrm{C}\right): \delta_{\mathrm{H}}=-2.86(\mathrm{~s}, \mathrm{br}$, $2 \mathrm{H},-\mathrm{NH}), 7.14(\mathrm{~s}, 3 \mathrm{H}$, Ar- $H), 7.39\left(\mathrm{t},{ }^{3} J_{\mathrm{H}-\mathrm{H}}=7.36 \mathrm{~Hz}, 2 \mathrm{H}\right.$, Ar- $\left.H\right), 7.49(\mathrm{~m}, 6 \mathrm{H}$, Ar- $H)$ $7.70\left(\mathrm{t},{ }^{3} J_{\mathrm{H}-\mathrm{H}}=7.35 \mathrm{~Hz}, 6 \mathrm{H}, \mathrm{Ar}-H\right), 8.07\left(\mathrm{~d},{ }^{3} J_{\mathrm{H}-\mathrm{H}}=7.35 \mathrm{~Hz}, 2 \mathrm{H}, \mathrm{Ar}-H\right), 8.52(\mathrm{~s}, 1 \mathrm{H}, \mathrm{Ar}-$ $H), 8.80\left(\mathrm{~m}, 4 \mathrm{H}, H_{\beta}\right), 8.89 \mathrm{ppm}\left(\mathrm{m}, 4 \mathrm{H}, H_{\beta}\right) ;{ }^{13} \mathrm{C} \mathrm{NMR}\left(100 \mathrm{MHz}, \mathrm{CDCl}_{3}\right): \delta=114.8$ $119.7,121.0,121.8,123.9,127.3,127.7,131.9,134.6,136.3,143.6,149.2,149.3,153.9$ ppm; UV/Vis (EtOAc): $\lambda_{\max }(\lg \varepsilon)=417$ (5.41), 514 (4.08), 547 (3.69), 591 (3.59), 648 nm (3.48); HRMS (MALDI): $m / z$ calcd for $\left[\mathrm{C}_{51} \mathrm{H}_{35} \mathrm{BrN}_{4} \mathrm{O}_{4}\right][\mathrm{M}+]$ : 846.1842 , found 846.1861 .

5,10,15,20-[3-(2-bromophenyl)benzoate]porphyrin 10b: Synthesized via Genera Procedure B using 2 (100 mg, $0.14 \mathrm{mmol})$, EDC (228 mg, $1.4 \mathrm{mmol}), \mathrm{N}$ hydroxysuccinimide $(170 \mathrm{mg}, 1.4 \mathrm{mmol}), \mathrm{K}_{2} \mathrm{CO}_{3}(203 \mathrm{mg}, 1.4 \mathrm{mmol})$ and 2 bromobenzoic acid $(338 \mathrm{mg}, 1.7 \mathrm{mmol})$. The reaction was completed after $6 \mathrm{~h}$. Yield: $83 \mathrm{mg}$ of purple crystals $(0.059 \mathrm{mmol}, 59 \%)$; m.p. $>300{ }^{\circ} \mathrm{C} ; R_{f}=0.85\left(\mathrm{CH}_{2} \mathrm{Cl}_{2} / n-\right.$ hexane/MeOH = 3:1:0.2, v/v/v); ${ }^{1} \mathrm{H}$ NMR $\left(400 \mathrm{MHz},\left(\mathrm{CD}_{3}\right)_{2} \mathrm{SO}, 25^{\circ} \mathrm{C}\right): \delta_{\mathrm{H}}=-2.95(2 \mathrm{H}$, $\mathrm{N} H), 7.51(\mathrm{~s}, 8 \mathrm{H}, \operatorname{Ar}-H), 7.78\left(\mathrm{~d},{ }^{3} J_{\mathrm{H}-\mathrm{H}}=7.77 \mathrm{~Hz}, 8 \mathrm{H}, \operatorname{Ar}-H\right), 7.91\left(\mathrm{t},{ }^{3} J_{\mathrm{H}-\mathrm{H}}=7.77 \mathrm{~Hz}\right.$ $5 \mathrm{H}, \operatorname{Ar}-H), 8.10(\mathrm{~m}, 4 \mathrm{H}, \mathrm{Ar}-H), 8.20(\mathrm{~m}, 7 \mathrm{H}, \mathrm{Ar}-H), 8.94 \mathrm{ppm}\left(\mathrm{s}, 8 \mathrm{H}, H_{\beta}\right) ;{ }^{13} \mathrm{C}$ NMR $\left(100 \mathrm{MHz}, \mathrm{CDCl}_{3}\right): \delta=119.4,121.3,122.0,128.1,128.4,128.7,131.7,132.1,132.7$, $134.3,134.6,142.9,149.4,164.9 \mathrm{ppm} ; \mathrm{UV} / \mathrm{Vis}(\mathrm{EtOAc}): \lambda_{\max }(\lg \varepsilon)=417(5.40), 514$ (4.08), 547 (3.68), 591 (3.59), $648 \mathrm{~nm}$ (3.49); HRMS (MALDI): $m / z$ calcd for $\left[\mathrm{C}_{72} \mathrm{H}_{42} \mathrm{Br}_{4} \mathrm{~N}_{4} \mathrm{O}_{8}\right][\mathrm{M}+]:$ 1405.9736, found 1405.9730

5,10,15,-Tris(3-hydroxyphenyl)-20-(3-phenylbenzoate)porphyrin 11a: The compound was synthesized using general procedure B: a $50 \mathrm{ml}$ round bottom flask containing $10 \mathrm{~mL}$ of DMF, $2(400 \mathrm{mg}, 0.59 \mathrm{mmol}), \mathrm{NaH}(14.2 \mathrm{mg}, 0.59 \mathrm{mmol})$, and benzoic anhydride $(133 \mathrm{mg}, 0.59 \mathrm{mmol})$. The reaction was complete after $1 \mathrm{~h}$. Yield: $170 \mathrm{mg}$ of purple crystals $(0.21 \mathrm{mmol}, 37 \%)$; m.p. $>300{ }^{\circ} \mathrm{C} ; R_{f}=0.34\left(\mathrm{CH}_{2} \mathrm{Cl}_{2} / n-\right.$ hexane $/ \mathrm{MeOH}=3: 1: 0.2, \mathrm{v} / \mathrm{v} / \mathrm{v}) ;{ }^{1} \mathrm{H}$ NMR $\left(400 \mathrm{MHz},\left(\mathrm{CD}_{3}\right)_{2} \mathrm{SO}, 25{ }^{\circ} \mathrm{C}\right): \delta_{\mathrm{H}}=-2.97(\mathrm{~s}$, $2 \mathrm{H}, \mathrm{N} H), 7.24(\mathrm{~m}, 3 \mathrm{H}, \mathrm{Ar}-H), 7.59(\mathrm{~m}, 11 \mathrm{H}, \mathrm{Ar}-H), 7.72\left(\mathrm{t},{ }^{3} J_{\mathrm{H}-\mathrm{H}}=7.37 \mathrm{~Hz}, 1 \mathrm{H}, \mathrm{Ar}-H\right)$, $7.77\left(\mathrm{~d},{ }^{3} J_{\mathrm{H}-\mathrm{H}}=7.67 \mathrm{~Hz}, 1 \mathrm{H}, \mathrm{Ar}-H\right), 7.89\left(\mathrm{t},{ }^{3} J_{\mathrm{H}-\mathrm{H}}=8.11 \mathrm{~Hz} 1 \mathrm{H}, \mathrm{Ar}-H\right), 8.21(\mathrm{~m}, 4 \mathrm{H}, \mathrm{Ar}-$ $H), 8.89\left(\mathrm{~m}, 8 \mathrm{H}, H_{\beta}\right), 9.88(\mathrm{~s}, \mathrm{br}, 3 \mathrm{H},-\mathrm{OH}) ;{ }^{13} \mathrm{C} \mathrm{NMR}\left(100 \mathrm{MHz},\left(\mathrm{CD}_{3}\right)_{2} \mathrm{SO}\right): \delta=115.6$ $118.9,120.5,120.6,122.3,125.7,126.3,128.3,128.6,129.3,129.4,130.3,131.9$ $132.1,142.8,149.7,156.2,165.3 \mathrm{ppm}$; UV/Vis (EtOAc): $\lambda_{\max }(\lg \varepsilon)=417(5.02), 514$ (3.76), 547 (3.50), 591 (3.13), $646 \mathrm{~nm}(2.96)$; HRMS (MALDI): $\mathrm{m} / \mathrm{z}$ calcd for $\left[\mathrm{C}_{51} \mathrm{H}_{34} \mathrm{~N}_{4} \mathrm{O}_{5}\right]\left[\mathrm{M}^{+}\right]:$782.2529, found 782.2531 .

5,10,15,20-Tetrakis[3-phenylbenzoate]porphyrin 11b: The compound was synthesized using general procedure $\mathrm{C}$ : a $50 \mathrm{ml}$ round bottom flask containing $10 \mathrm{~mL}$ of DMF, 2 (100 mg, $0.14 \mathrm{mmol}), \mathrm{K}_{2} \mathrm{CO}_{3}(204 \mathrm{mg}, 1.4 \mathrm{mmol})$, and benzoic anhydride (400 $\mathrm{mg}, 1.77 \mathrm{mmol})$. The reaction was complete after $7 \mathrm{~h}$. Yield: $148 \mathrm{mg}$ of purple crystals $(0.13 \mathrm{mmol}, 92 \%)$; m.p. $>300{ }^{\circ} \mathrm{C} ; R_{f}=0.9\left(\mathrm{CH}_{2} \mathrm{Cl}_{2} / n\right.$-hexane $\left./ \mathrm{MeOH}=3: 1: 0.2, \mathrm{v} / \mathrm{v} / \mathrm{v}\right)$ ${ }^{1} \mathrm{H}$ NMR $\left(400 \mathrm{MHz}, \mathrm{CDCl}_{3}, 25^{\circ} \mathrm{C}\right): \delta_{\mathrm{H}}=-2.74(\mathrm{~s}, 2 \mathrm{H}), 7.54\left(\mathrm{t},{ }^{3} J_{\mathrm{H}-\mathrm{H}}=7.4 \mathrm{~Hz}, 8 \mathrm{H}, \mathrm{Ar}-\right.$ $\left.H_{m}\right), 7.64\left(\mathrm{t},{ }^{3} J_{\mathrm{H}-\mathrm{H}}=7.1 \mathrm{~Hz}, 4 \mathrm{H}, \mathrm{Ar}-H_{m}\right), 7.72\left(\mathrm{~d},{ }^{3} J_{\mathrm{H}-\mathrm{H}}=7.8 \mathrm{~Hz}, 4 \mathrm{H}, \mathrm{Ar}-H\right), 7.86\left(\mathrm{t},{ }^{3} J_{\mathrm{H}-\mathrm{H}}\right.$ $=7.7 \mathrm{~Hz}, 4 \mathrm{H}, \mathrm{Ar}-H), 8.20(\mathrm{~m}, 8 \mathrm{H}, \mathrm{Ar}-H), 8.34\left(\mathrm{~d},{ }^{3} J_{\mathrm{H}-\mathrm{H}}=7.7 \mathrm{~Hz}, 8 \mathrm{H}, \mathrm{Ar}-\mathrm{CH}\right), 9.01 \mathrm{ppm}$ $\left(\mathrm{s}, 8 \mathrm{H}, H_{\beta}\right) ;{ }^{13} \mathrm{C}$ NMR $\left(100 \mathrm{MHz}, \mathrm{CDCl}_{3}\right): \delta=119.1,121.3,127.8,128.1,128.6,129.6$ $130.3,132.4,133.7,143.5,149.6,165.4 \mathrm{ppm} ; \mathrm{UV} / \mathrm{Vis}$ (EtOAc): $\lambda_{\max }(\lg \varepsilon)=417$ (5.02), 515 (3.76), $547(3.51), 590$ (3.15), $646 \mathrm{~nm}$ (2.97); HRMS (MALDI): $\mathrm{m} / z$ calcd for $\left[\mathrm{C}_{72} \mathrm{H}_{46} \mathrm{~N}_{4} \mathrm{O}_{8}\right]\left[\mathrm{M}^{+}\right]:$1094.3316, found 1094.3311. 
5,10,15-Tris(3-hydroxyphenyl)-20-(3-trifluoromethanesulfonatephenyl)porphyrin 12a: Synthesized via General Procedure B using 2 (400 $\mathrm{mg}, 0.59 \mathrm{mmol})$, pyridine $(0.046 \mathrm{~mL}, 0.59 \mathrm{mmol})$, and triflate $(0.12 \mathrm{~mL}, 1.2 \mathrm{mmol})$. The reaction was completed after 1 hour. Yield: $177 \mathrm{mg}$ of purple crystals $(0.22 \mathrm{mmol}, 37 \%)$; m.p. $>300{ }^{\circ} \mathrm{C} ; R_{f}=$ $0.38\left(\mathrm{CH}_{2} \mathrm{Cl}_{2} / n\right.$-hexane/MeOH $\left.=3: 1: 0.2, \mathrm{v} / \mathrm{v} / \mathrm{v}\right) ;{ }^{1} \mathrm{H}$ NMR $\left(400 \mathrm{MHz},\left(\mathrm{CD}_{3}\right)_{2} \mathrm{SO}, 25^{\circ} \mathrm{C}\right)$ $\delta_{\mathrm{H}}=-2.99(\mathrm{~s}, 2 \mathrm{H}, \mathrm{N} H), 7.22(\mathrm{~m}, 4 \mathrm{H}, \mathrm{o}-\mathrm{Ar}-H), 7.59(\mathrm{~m}, 12 \mathrm{H}, \mathrm{Ar}-H), 8.86\left(\mathrm{~s}, 8 \mathrm{H}, H_{\beta}\right)$, $9.87(\mathrm{~s}, 3 \mathrm{H}, \mathrm{OH}) ;{ }^{19} \mathrm{~F}$ NMR $\left(400 \mathrm{MHz}, \mathrm{CDCl}_{3}, 25{ }^{\circ} \mathrm{C}\right): \delta=-39.42(\mathrm{~s}) \mathrm{ppm} ;{ }^{13} \mathrm{C} \mathrm{NMR}$ $\left(100 \mathrm{MHz},\left(\mathrm{CD}_{3}\right)_{2} \mathrm{SO}, 25^{\circ} \mathrm{C}\right): 115.5,120.3,122.3,126.3,128.3,142.8,156.2 ; \mathrm{UV} / \mathrm{Vi}$ (EtOAc): $\lambda_{\max }(\lg \varepsilon)=417$ (5.04),513 (3.71), 547 (3.32), 591(3.21), $647 \mathrm{~nm}$ (3.03); HRMS (MALDI): $m / z$ calcd for $\left[\mathrm{C}_{45} \mathrm{H}_{29} \mathrm{~F}_{3} \mathrm{~N}_{4} \mathrm{O}_{6} \mathrm{~S}\right]\left[\mathrm{M}^{+}\right]: 810.1760$, found 810.1751 .

\section{5,10,15,20-Tetrakis(3-(trifluoromethanesulfonate)phenyl)porphyrin}

12b:

Synthesized via General Procedure C using $2(400 \mathrm{mg}, 0.59 \mathrm{mmol})$, pyridine $(0.46 \mathrm{~mL}$, $5.9 \mathrm{mmol})$, and trifluoromethanesulfonyl chloride $(0.63 \mathrm{~mL}, 5.9 \mathrm{mmol})$. The reaction was completed after 8 hour. Yield: $655 \mathrm{mg}$ of purple crystals $(0.54 \mathrm{mmol}, 92 \%)$; m.p. $>300{ }^{\circ} \mathrm{C}, R_{f}=0.95\left(\mathrm{CH}_{2} \mathrm{Cl}_{2} / n\right.$-hexane $\left./ \mathrm{MeOH}=3: 1: 0.2, \mathrm{v} / \mathrm{v} / \mathrm{v}\right) ;{ }^{1} \mathrm{H}$ NMR $(400 \mathrm{MHz}$ $\left.\mathrm{CDCl}_{3}, 25{ }^{\circ} \mathrm{C}\right): \delta_{\mathrm{H}}=-2.79(2 \mathrm{H}, \mathrm{N} H), 7.79\left(\mathrm{~d},{ }^{3} J_{\mathrm{H}-\mathrm{H}}=8 \mathrm{~Hz}, 4 \mathrm{H}, \mathrm{Ar}-H\right), 7.90\left(\mathrm{t},{ }^{3} J_{\mathrm{H}-\mathrm{H}}=\right.$ $7.65 \mathrm{~Hz}, 4 \mathrm{H}, \operatorname{Ar}-H), 8.25$ (s, 4H, $o-\operatorname{Ar}-H), 8.31(\mathrm{~m}, 4 \mathrm{H}, \operatorname{Ar}-H), 8.93 \mathrm{ppm}\left(\mathrm{s}, 8 \mathrm{H}, H_{\beta}\right)$; ${ }^{13} \mathrm{C}$ NMR $\left(150 \mathrm{MHz}, \mathrm{CDCl}_{3}\right): \delta=114.18,117.37,118.19,120.56,121.12,123.75$ 127.23, 128.63, 134.42, 134.47, 144.24, $148.35 \mathrm{ppm} ;{ }^{19} \mathrm{~F}$ NMR $\left(400 \mathrm{MHz}, \mathrm{CDCl}_{3}, 2\right.$ $\left.{ }^{\mathrm{C}}\right): \delta=-72.55 \mathrm{ppm}\left(\mathrm{CF}_{3}\right) ; \mathrm{UV} / \mathrm{Vis}(\mathrm{EtOAc}): \lambda_{\max }(\lg \varepsilon)=415$ (5.40), 512 (4.10), 545 (3.63), 589 (3.60), $645 \mathrm{~nm}$ (3.24); HRMS (MALDI): $\mathrm{m} / z$ calcd for $\left[\mathrm{C}_{48} \mathrm{H}_{26} \mathrm{~F}_{12} \mathrm{~N}_{4} \mathrm{O}_{12} \mathrm{~S}_{4}\right]$ $\left[\mathrm{M}^{+}\right]$: 1206.0238 , found 1206.0226 .

5,10,15-Tris(3-hydroxyphenyl)-20-[3-(4-methylbenzenesulfonate)phenyl]porphyrin 13a: Synthesized via General Procedure B using 2 ( $400 \mathrm{mg}, 0.59 \mathrm{mmol}$ ), $\mathrm{K}_{2} \mathrm{CO}_{3}(244$ $\mathrm{mg}, 1.8 \mathrm{mmol}$ ), and 4-toluenesulfonyl chloride (448 mg, $2.3 \mathrm{mmol})$. The reaction was completed after $2 \mathrm{~h}$. Yield: $171 \mathrm{mg}$ of purple crystals $(0.20 \mathrm{mmol}, 35 \%)$; m.p. $>300{ }^{\circ} \mathrm{C}$ $R_{f}=0.29\left(\mathrm{CH}_{2} \mathrm{Cl}_{2} / n\right.$-hexane/MeOH $\left.=3: 1: 0.2, \mathrm{v} / \mathrm{v} / \mathrm{v}\right) ;{ }^{1} \mathrm{H}$ NMR $\left(400 \mathrm{MHz}, \mathrm{CDCl}_{3}, 25\right.$ $\left.{ }^{\circ} \mathrm{C}\right): \delta_{\mathrm{H}}=-2.92(2 \mathrm{H}, \mathrm{N} H), 2.2\left(\mathrm{~s}, 3 \mathrm{H},-\mathrm{CH}_{3}\right), 7.51(\mathrm{~m}, 2 \mathrm{H}, \mathrm{Ar}-\mathrm{H}), 7.59(\mathrm{~m}, 4 \mathrm{H}, \mathrm{Ar}-\mathrm{H})$ $7.67(\mathrm{~m}, 6 \mathrm{H}, \operatorname{Ar}-H), 7.76(\mathrm{~m}, 4 \mathrm{H}, \mathrm{Ar}-H) 7.85\left(\mathrm{~d},{ }^{3} J_{\mathrm{H}-\mathrm{H}}=8 \mathrm{~Hz}, 4 \mathrm{H}, \operatorname{Ar}-H\right) 8.06\left(\mathrm{~d},{ }^{3} J_{\mathrm{H}-\mathrm{H}}\right.$ $\left.=4 \mathrm{~Hz}, 2 \mathrm{H}, H_{\beta}\right) 8.56\left(\mathrm{~m},{ }^{3} J_{\mathrm{H}-\mathrm{H}}=4 \mathrm{~Hz}, 2 \mathrm{H}, H_{\beta}\right) 8.83 \mathrm{ppm}\left(\mathrm{d},{ }^{3} J_{\mathrm{H}-\mathrm{H}}=4 \mathrm{~Hz}, 4 \mathrm{H}, H_{\beta}\right) ;{ }^{13} \mathrm{C}$ NMR $\left(150 \mathrm{MHz}, \mathrm{CDCl}_{3}\right) 21.5,115.5,119.1,120.4,120.5,122.3,124.3,125.7,126.2$ $128.2,128.3,128.4,128.6,129.3,142.6,142.8,150.0,150.4,154.7,156.2 \mathrm{ppm}$ UV/Vis (EtOAc): $\lambda_{\max }(\lg \varepsilon)=417(5.42), 514(4.10), 547(3.65), 591(3.60), 649 \mathrm{~nm}$ (3.25); HRMS (MALDI): $\mathrm{m} / z$ calcd for $\left[\mathrm{C}_{51} \mathrm{H}_{36} \mathrm{~N}_{4} \mathrm{O}_{6} \mathrm{~S}\right]\left[\mathrm{M}^{+}\right]: 832.2356$, found 832.2348

\section{5,10,15,20-Tetrakis[3-(4-methylbenzenesulfonate)phenyl]porphyrin}

13b:

Synthesized via General Procedure C using $2(400 \mathrm{mg}, 0.59 \mathrm{mmol})$, pyridine $(0.46 \mathrm{~mL}$, $5.9 \mathrm{mmol})$, and 4-toluenesulfonyl chloride $(1.12 \mathrm{~g}, 5.9 \mathrm{mmol})$. The reaction was completed after $4 \mathrm{~h}$. Yield: $687 \mathrm{mg}$ of purple crystals $(0.53 \mathrm{mmol}, 90 \%)$; m.p. $>300{ }^{\circ} \mathrm{C}$, $R_{f}=0.91\left(\mathrm{CH}_{2} \mathrm{Cl}_{2} / n\right.$-hexane $\left./ \mathrm{MeOH}=3: 1: 0.2, \mathrm{v} / \mathrm{v} / \mathrm{v}\right) ;{ }^{1} \mathrm{H}$ NMR $\left(400 \mathrm{MHz}, \mathrm{CDCl}_{3}, 25\right.$ $\left.{ }^{\circ} \mathrm{C}\right): \delta_{\mathrm{H}}=-3.04(\mathrm{~s}, 2 \mathrm{H}, \mathrm{NH}), 2.31\left(\mathrm{~s}, 12 \mathrm{H}, \mathrm{C} H_{3}\right), 7.32\left(\mathrm{~d},{ }^{3} J_{\mathrm{H}-\mathrm{H}}=7.87 \mathrm{~Hz}, 8 \mathrm{H}, \mathrm{Ar}-\mathrm{H}\right)$ $7.56(\mathrm{~m}, 4 \mathrm{H}, \mathrm{Ar}-H), 7.72\left(\mathrm{t},{ }^{3} J_{\mathrm{H}-\mathrm{H}}=7.87 \mathrm{~Hz}, 5 \mathrm{H}, \mathrm{Ar}-H\right), 7.79(\mathrm{~m}, 3 \mathrm{H}, \mathrm{Ar}-H), 7.89(\mathrm{~d}$ $\left.{ }^{3} J_{\mathrm{H}-\mathrm{H}}=7.87 \mathrm{~Hz}, 8 \mathrm{H}, \mathrm{Ar}-H\right), 8.06(\mathrm{~m}, 4 \mathrm{H}, \mathrm{Ar}-H), 8.64 \mathrm{ppm}\left(\mathrm{s}, 8 \mathrm{H}, H_{\beta}\right) ;{ }^{13} \mathrm{C}$ NMR $(150$ $\left.\mathrm{MHz}, \mathrm{CDCl}_{3}\right): \delta=21.6,118.5,122.2,127.9,128.2,128.6,130.0,132.4,133.2,143.4$, 145.6, $148.2 \mathrm{ppm}$; UV/Vis (EtOAc): $\lambda_{\max }(\lg \varepsilon)=416$ (5.44), 513 (4.11), $546(3.63), 590$ (3.59), $646 \mathrm{~nm}$ (3.23); HRMS (MALDI): $\mathrm{m} / z$ calcd for $\left[\mathrm{C}_{72} \mathrm{H}_{54} \mathrm{~N}_{4} \mathrm{O}_{12} \mathrm{~S}_{4}\right]\left[\mathrm{M}^{+}\right]$: 1294.2621, found 1294.2590 .

[5,10,15-Tris(3-hydroxyphenyl)-20-[3-(phenoxy)phenyl]porphyrinato]zinc(II) 15: Synthesized via General Procedure D using 14 (50 mg, $0.067 \mathrm{mmol}), \mathrm{Cu}(\mathrm{II}) \mathrm{OAc}$ (19.98 $\mathrm{mg}, 0.11 \mathrm{mmol})$, phenylboronic acid $(26.99 \mathrm{mg}, 0.22 \mathrm{mmol})$ and pyridine $(0.2 \mathrm{~mL})$ in dioxane $(10 \mathrm{~mL})$. The reaction was completed after $12 \mathrm{~h}$. Yield: $22 \mathrm{mg}$ of purple crystals $(0.026 \mathrm{mmol}, 39 \%)$; m.p. $>300{ }^{\circ} \mathrm{C}, R_{f}=0.31\left(\mathrm{CH}_{2} \mathrm{Cl}_{2} / n\right.$-hexane $/ \mathrm{MeOH}=$ $3: 1: 0.2, \mathrm{v} / \mathrm{v} / \mathrm{v}) ;{ }^{1} \mathrm{H}$ NMR $\left(400 \mathrm{MHz},\left(\mathrm{CD}_{3}\right)_{2} \mathrm{SO}, 25{ }^{\circ} \mathrm{C}\right): \delta_{\mathrm{H}}=7.09\left(\mathrm{t},{ }^{3} J_{\mathrm{H}-\mathrm{H}}=7.46 \mathrm{~Hz}\right.$ $1 \mathrm{H}, \operatorname{Ar}-H), 7.15(\mathrm{~m}, 3 \mathrm{H}, \mathrm{Ar}-H), 7.28\left(\mathrm{~d},{ }^{3} J_{\mathrm{H}-\mathrm{H}}=7.90 \mathrm{~Hz}, 2 \mathrm{H}, \mathrm{Ar}-H\right), 7.41\left(\mathrm{t},{ }^{3} J_{\mathrm{H}-\mathrm{H}}=7.46\right.$ $\mathrm{Hz}, 2 \mathrm{H}, \mathrm{Ar}-H) 7.45(\mathrm{~m}, 1 \mathrm{H}, \mathrm{Ar}-H), 7.54(\mathrm{~m}, 9 \mathrm{H}, \mathrm{Ar}-H), 7.69(\mathrm{~m}, 1 \mathrm{H}, \mathrm{Ar}-H), 7.76(\mathrm{t}$ $\left.{ }^{3} J_{\mathrm{H}-\mathrm{H}}=7.76 \mathrm{~Hz}, 1 \mathrm{H}, \mathrm{Ar}-H\right), 7.91\left(\mathrm{~d},{ }^{3} J_{\mathrm{H}-\mathrm{H}}=7.33 \mathrm{~Hz}, 1 \mathrm{H}, \mathrm{Ar}-H\right), 8.79\left(\mathrm{~d},{ }^{3} J_{\mathrm{H}-\mathrm{H}}=3.54\right.$ $\left.\mathrm{Hz}, 6 \mathrm{H}, H_{\beta}\right), 8.80\left(\mathrm{~d},{ }^{3} J_{\mathrm{H}-\mathrm{H}}=4.70 \mathrm{~Hz}, 6 \mathrm{H}, H_{\beta}\right), 9.75(\mathrm{~s}, 3 \mathrm{H},-\mathrm{O} H) ;{ }^{13} \mathrm{C} \mathrm{NMR}(100 \mathrm{MHz}$ $\left.\mathrm{CDCl}_{3}, 25^{\circ} \mathrm{C}\right): \delta=114.3,115.3,117.9,118.8,120.7,122.0,123.2,127.3,127.4,127.5$, $127.7,129.6,129.7,129.8,131.5,131.6,131.7,133.7,136.6,145.4 \mathrm{ppm} ; \mathrm{UV} / \mathrm{Vis}$ (EtOAc): $\lambda_{\max }(\lg \varepsilon)=421(6.83), 553(5.40), 5.93 \mathrm{~nm}(4.78)$; HRMS (MALDI): $\mathrm{m} / \mathrm{z}$ calcd for $\left[\mathrm{C}_{50} \mathrm{H}_{34} \mathrm{~N}_{4} \mathrm{O}_{4} \mathrm{Zn}\right]\left[\mathrm{M}^{+}\right]: 816.1715$, found 816.1710 .

\section{[5-(3-(3-cyanophenoxy)phenyl)-10,15,20-tris(3-}

hydroxyphenyl)porphyrinato]zinc(II) 16: Synthesized via General Procedure D using $14(50 \mathrm{mg}, 0.067 \mathrm{mmol}), \mathrm{Cu}(\mathrm{II}) \mathrm{OAc}(19.98 \mathrm{mg}, 0.11 \mathrm{mmol}), 3$-cyanophenylboronic acid $(26.99 \mathrm{mg}, 0.22 \mathrm{mmol})$ and pyridine $(0.2 \mathrm{~mL})$ in dioxane $(10 \mathrm{~mL})$. The reaction was completed after $15 \mathrm{~h}$. Yield: $19.7 \mathrm{mg}$ of purple crystals $(0.023 \mathrm{mmol}, 35 \%)$ m.p. $>300{ }^{\circ} \mathrm{C}, R_{f}=0.31\left(\mathrm{CH}_{2} \mathrm{Cl}_{2} / n\right.$-hexane/MeOH $\left.=3: 1: 0.2, \mathrm{v} / \mathrm{v} / \mathrm{v}\right) ;{ }^{1} \mathrm{H}$ NMR $(400 \mathrm{MHz}$, $\left.\left(\mathrm{CD}_{3}\right)_{2} \mathrm{SO}, 25^{\circ} \mathrm{C}\right): \delta_{\mathrm{H}}=7.17(\mathrm{~m}, 3 \mathrm{H}, \mathrm{Ar}-H), 7.55(\mathrm{~m}, 12 \mathrm{H}, \mathrm{Ar}-H), 7.64(\mathrm{~m}, 2 \mathrm{H}, \mathrm{Ar}-H)$, $7.81(\mathrm{~m}, 2 \mathrm{H}, \operatorname{Ar}-H), 8.01(\mathrm{~m}, 1 \mathrm{H}, \operatorname{Ar}-H), 8.80\left(\mathrm{~s}, 4 \mathrm{H}, H_{\beta}\right) 8.83\left(\mathrm{~s}, 4 \mathrm{H}, H_{\beta}\right), 9.77(\mathrm{~s}, 3 \mathrm{H}$, $\mathrm{O} H) ;{ }^{13} \mathrm{C}$ NMR $\left(100 \mathrm{MHz}, \mathrm{CDCl}_{3}, 25{ }^{\circ} \mathrm{C}\right): \delta=112.8,114.9,118.9,120.6,120.62$, $120.65,120.7,122.3,126.2,127.8,131.7,131.9,144.4,149.4,149.5,149.6,149.9$, $155.9 \mathrm{ppm} ; \quad \mathrm{UV} / \mathrm{Vis}(\mathrm{EtOAc}): \lambda_{\max }(\lg \varepsilon)=421$ (6.81), 552 (4.92), $5.94 \mathrm{~nm}(4.39)$ HRMS (MALDI): $m / z$ calcd for $\left[\mathrm{C}_{51} \mathrm{H}_{33} \mathrm{~N}_{5} \mathrm{O}_{4} \mathrm{Zn}\right]\left[\mathrm{M}^{+}\right]: 841.1668$, found 841.1665.

\section{[5,10,15-Tris(3-hydroxyphenyl)-20-[3-(3-}

methoxyphenyl)phenyl]porphyrinato]zinc(II) 17: Synthesized via General Procedure D using $14(70 \mathrm{mg}, 0.095 \mathrm{mmol}), \mathrm{Cu}(\mathrm{II}) \mathrm{OAc}(27.2 \mathrm{mg}, 0.15 \mathrm{mmol})$, and $3-$ methoxyphenylboronic acid $(44 \mathrm{mg}, 0.29 \mathrm{mmol})$. The reaction was completed after $2 \mathrm{~h}$ Yield: $30 \mathrm{mg}$ of purple crystals $(0.035 \mathrm{mmol}, 37 \%)$; m.p. $>300{ }^{\circ} \mathrm{C}, R_{f}=0.29\left(\mathrm{CH}_{2} \mathrm{Cl}_{2} / n-\right.$ hexane/MeOH $=3: 1: 0.2, \mathrm{v} / \mathrm{v} / \mathrm{v}) ;{ }^{1} \mathrm{H}$ NMR $\left(400 \mathrm{MHz},\left(\mathrm{CD}_{3}\right)_{2} \mathrm{SO}, 25^{\circ} \mathrm{C}\right): \delta_{\mathrm{H}}=3.76(\mathrm{~s}$ $\left.3 \mathrm{H},-\mathrm{OCH} H_{3}\right), 6.70\left(\mathrm{~d},{ }^{3} J_{\mathrm{H}-\mathrm{H}}=8.01 \mathrm{~Hz}, 1 \mathrm{H}, \mathrm{Ar}-H\right), 6.87\left(\mathrm{~d},{ }^{3} J_{\mathrm{H}-\mathrm{H}}=8.01 \mathrm{~Hz}, 2 \mathrm{H}, \mathrm{Ar}-H\right)$ $7.21\left(\mathrm{~d},{ }^{3} J_{\mathrm{H}-\mathrm{H}}=8.01 \mathrm{~Hz}, 3 \mathrm{H}, \mathrm{Ar}-H\right), 7.34\left(\mathrm{t},{ }^{3} J_{\mathrm{H}-\mathrm{H}}=8.01 \mathrm{~Hz}, 1 \mathrm{H}, \mathrm{Ar}-H\right), 7.50\left(\mathrm{dd},{ }^{3} J_{\mathrm{H}-\mathrm{H}}\right.$
$=8.01 \mathrm{~Hz}, 1 \mathrm{H}, \operatorname{Ar}-H), 7.58(\mathrm{~m}, 9 \mathrm{H}, \operatorname{Ar}-H), 7.75(\mathrm{~s}, 1 \mathrm{H}, \operatorname{Ar}-H), 7.80\left(\mathrm{t},{ }^{3} J_{\mathrm{H}-\mathrm{H}}=8.01 \mathrm{~Hz}\right.$, $1 \mathrm{H}, \operatorname{Ar}-H), 7.96\left(\mathrm{~d},{ }^{3} J_{\mathrm{H}-\mathrm{H}}=7.12 \mathrm{~Hz}, 1 \mathrm{H}\right), 8.84\left(\mathrm{~d},{ }^{3} J_{\mathrm{H}-\mathrm{H}}=4.32 \mathrm{~Hz}, 6 \mathrm{H}, H_{\beta}\right), 8.86\left(\mathrm{~d},{ }^{3} J_{\mathrm{H}-}\right.$ $\left.\mathrm{H}=4.71 \mathrm{~Hz}, 2 \mathrm{H}, H_{\beta}\right), 9.80(\mathrm{~s}, 3 \mathrm{H},-\mathrm{OH}) ;{ }^{13} \mathrm{C} \mathrm{NMR}\left(100 \mathrm{MHz}, \mathrm{CDCl}_{3}, 25^{\circ} \mathrm{C}\right): \delta=55.7$ $105.4,109.8,111.3,114.9,119.4,120.8,120.9,122.3,126.2,127.8,131.0,131.6$ $131.9,132.1,144.3,145.0,149.4,149.5,149.6,149.7,155.3,155.9,161.2 \mathrm{ppm}$; UV/Vis (EtOAc): $\lambda_{\max }(\lg \varepsilon)=421$ (6.85), 553 (5.40), $593 \mathrm{~nm}$ (4.78); HRMS (MALDI): $m / z$ calcd for $\left[\mathrm{C}_{51} \mathrm{H}_{36} \mathrm{~N}_{4} \mathrm{O}_{5} \mathrm{Zn}\right]\left[\mathrm{M}^{+}\right]$: 846.1821, found 846.1819.

5,10,15,20-tetrakis[3-(4-tert-butylbenzyloxy)phenyl]porphyrin 18: Synthesized via General Procedure C using 2 ( $400 \mathrm{mg}, 0.59 \mathrm{mmol}), \mathrm{K}_{2} \mathrm{CO}_{3}(815 \mathrm{mg}, 6 \mathrm{mmol})$, and 4 tertbutylbenzylbromide $(1.4 \mathrm{~mL}, 12 \mathrm{mmol})$. The reaction was complete after $12 \mathrm{~h}$ Yield: $707 \mathrm{mg}$ of purple crystals $(0.56 \mathrm{mmol}, 96 \%)$; m.p. $>300{ }^{\circ} \mathrm{C} ; R_{f}=0.95\left(\mathrm{CH}_{2} \mathrm{Cl}_{2} / n-\right.$ hexane $/ \mathrm{MeOH}=3: 1: 0.2, \mathrm{v} / \mathrm{v} / \mathrm{v}) ;{ }^{1} \mathrm{H}$ NMR $\left(400 \mathrm{MHz}, \mathrm{CDCl}_{3}, 25^{\circ} \mathrm{C}\right): \delta_{\mathrm{H}}=-2.70(\mathrm{~s}, 2 \mathrm{H}$ $\mathrm{N} H), 1.39(\mathrm{~s}, 36 \mathrm{H}, t \mathrm{Bu}-H), 5.28\left(\mathrm{~s}, 8 \mathrm{H},-\mathrm{CH}_{2}\right), 7.49(\mathrm{~m}, 20 \mathrm{H}, t \mathrm{Bu}-\mathrm{Ar}-H / \mathrm{Ar}-H), 7.70(\mathrm{t}$, $\left.{ }^{3} J_{\mathrm{H}-\mathrm{H}}=7.76 \mathrm{~Hz}, 4 \mathrm{H}, \mathrm{Ph}-H_{m}\right), 7.89\left(\mathrm{~d},{ }^{3} J_{\mathrm{H}-\mathrm{H}}=7.24 \mathrm{~Hz}, 4 \mathrm{H}, \operatorname{Ar}-H\right), 7.95(\mathrm{~s}, 4 \mathrm{H}, \mathrm{Ar}-H)$, $8.94 \mathrm{ppm}\left(\mathrm{s}, 8 \mathrm{H}, H_{\mathrm{\beta}}\right) ;{ }^{13} \mathrm{C} \mathrm{NMR}\left(100 \mathrm{MHz}, \mathrm{CDCl}_{3}\right): \delta=27.0,31.4,70.2,114.7,119.9$, 121.4, 125.6, 127.6, 127.7, 127.9, 133.9, 143.5, 151.1, $157.3 \mathrm{ppm} ; \mathrm{UV} / \mathrm{Vis}$ (EtOAc): $\lambda_{\max }(\lg \varepsilon)=417$ (5.02), 515 (3.76), 547 (3.50), 591 (3.16), $645 \mathrm{~nm}$ (2.98); HRMS (MALDI): $m / z$ calcd for $\left[\mathrm{C}_{88} \mathrm{H}_{76} \mathrm{~N}_{4} \mathrm{O}_{4}\right]\left[\mathrm{M}^{+}\right]: 1262.6649$, found 1262.6635

5,10,15,20-Tetrakis[3-(hexyloxy)phenyl]porphyrin 19: Synthesized via General Procedure $\mathrm{C}$ using 2 (400 mg, $0.59 \mathrm{mmol}), \mathrm{K}_{2} \mathrm{CO}_{3}(815 \mathrm{mg}, 5.9 \mathrm{mmol})$, and 1iodohexane $(1.4 \mathrm{~mL}, 5.9 \mathrm{mmol})$. The reaction was complete after $5 \mathrm{~h}$. Yield: $569 \mathrm{mg}$ of purple crystals $(0.56 \mathrm{mmol}, 94 \%)$; m.p. $>300{ }^{\circ} \mathrm{C} ; R_{f}=0.9\left(\mathrm{CH}_{2} \mathrm{Cl}_{2} / n\right.$-hexane $/ \mathrm{MeOH}=$ $3: 1: 0.2, \mathrm{v} / \mathrm{v} / \mathrm{v}) ;{ }^{1} \mathrm{H}$ NMR $\left(400 \mathrm{MHz}, \mathrm{CDCl}_{3}, 25{ }^{\circ} \mathrm{C}\right): \delta_{\mathrm{H}}=-2.81(\mathrm{~s}, 2 \mathrm{H}, \mathrm{N} H), 0.88(\mathrm{~m}$, $28 \mathrm{H}$, alkyl- $H), 1.55(\mathrm{~m}, 6 \mathrm{H}$, , alkyl- $H), 1.87\left(\mathrm{q},{ }^{3} J_{\mathrm{H}-\mathrm{H}}=6.76 \mathrm{~Hz}, 8 \mathrm{H},-\mathrm{CH}_{2}\right), 4.14\left(\mathrm{t},{ }^{3} J_{\mathrm{H}-\mathrm{H}}\right.$ $\left.=6.66 \mathrm{~Hz}, 8 \mathrm{H},-\mathrm{OCH}_{2}\right) 8.88\left(\mathrm{~d},{ }^{3} \mathrm{~J}_{\mathrm{H}-\mathrm{H}}=4.64 \mathrm{~Hz}, 8 \mathrm{H}, H_{\beta}\right) ;{ }^{13} \mathrm{C} \mathrm{NMR}\left(100 \mathrm{MHz}, \mathrm{CDCl}_{3}\right)$ : $\delta=22.6,25.7,29.4,29.7,31.6,68.2,114.2,119.9,121.0,127.4,127.5,143.4,157.5$ $\mathrm{ppm}$; UV/Vis (EtOAc): $\lambda_{\max }(\lg \varepsilon)=417$ (5.04), 515 (3.75), 547 (3.50), 589 (3.10), 648 nm (2.97); HRMS (MALDI): $m / z$ calcd for $\left[\mathrm{C}_{68} \mathrm{H}_{78} \mathrm{~N}_{4} \mathrm{O}_{4}\right]\left[\mathrm{M}^{+}\right]$: 1014.6023, found 1014.602 .

5-(3-Hydroxyphenyl)-10,15,20-tris[3-(4-tert-butylbenzyloxy)phenyl]porphyrin 20: Synthesized via General Procedure $\mathrm{C}$ using $2(400 \mathrm{mg}, 0.59 \mathrm{mmol}), \mathrm{K}_{2} \mathrm{CO}_{3}(800 \mathrm{mg}, 6$ $\mathrm{mmol})$, and 4-tert-butylbenzylbromide $(1.4 \mathrm{~mL}, 6 \mathrm{mmol})$. The reaction was complete after 7 h. Yield: $382 \mathrm{mg}(0.34 \mathrm{mmol}, 37 \%)$; m.p. $>300{ }^{\circ} \mathrm{C}, R_{f}=0.82\left(\mathrm{CH}_{2} \mathrm{Cl}_{2} / n\right.$ hexane $/ \mathrm{MeOH}=3: 1: 0.2, \mathrm{v} / \mathrm{v} / \mathrm{v}) ;{ }^{1} \mathrm{H}$ NMR $\left(400 \mathrm{MHz}, \mathrm{CDCl}_{3}, 25{ }^{\circ} \mathrm{C}\right): \delta_{\mathrm{H}}=-2.68(\mathrm{~s}, \mathrm{br}$, $2 \mathrm{H}, \mathrm{N} H), 1.40(\mathrm{~s}, 27 \mathrm{H}, t-\mathrm{Bu}-H), 5.27(\mathrm{~s}, 6 \mathrm{H}, \mathrm{CH}), 7.48\left(\mathrm{q},{ }^{3} J_{\mathrm{H}-\mathrm{H}}=3.64 \mathrm{~Hz}, 18 \mathrm{H}, t-\mathrm{BU}-\right.$ $\operatorname{Ar} H, \operatorname{Ar}-H), 7.69\left(\mathrm{q},{ }^{3} J_{\mathrm{H}-\mathrm{H}}=8.00 \mathrm{~Hz}, 3 \mathrm{H}, \operatorname{Ar}-H\right), 7.78\left(\mathrm{~d},{ }^{3} J_{\mathrm{H}-\mathrm{H}}=7.4 \mathrm{~Hz}, 1 \mathrm{H}, \operatorname{Ar}-H\right)$ $7.90\left(\mathrm{t},{ }^{3} J_{\mathrm{H}-\mathrm{H}}=7.12 \mathrm{~Hz}, 3 \mathrm{H}, \mathrm{Ar}-H\right), 7.97(\mathrm{~s}, \mathrm{br}, 3 \mathrm{H}, \mathrm{Ar}-H), 8.90\left(\mathrm{~d},{ }^{3} J_{\mathrm{H}-\mathrm{H}}=4.64 \mathrm{~Hz}, 2 \mathrm{H}\right.$, $\left.H_{\beta}\right), 8.94 \mathrm{ppm}\left(\mathrm{d},{ }^{3} J_{\mathrm{H}-\mathrm{H}}=8.36 \mathrm{~Hz}, 6 \mathrm{H}, H_{\beta}\right) ;{ }^{13} \mathrm{C} \mathrm{NMR}\left(100 \mathrm{MHz}, \mathrm{CDCl}_{3}\right): \delta=31.4$, $34.6,70.2,114.7,114.8,119.6,119.9,120.0,121.5,121.8,125.6,127.7,133.9,133.91$, 143.4, 143.5, 143.6, 151.1, 153.8, $157.3 \mathrm{ppm}$; UV/Vis (EtOAc): $\lambda_{\max }(\lg \varepsilon)=417$ (5.02), 515 (3.75), 548 (3.51), 590 (3.15), $647 \mathrm{~nm}$ (2.99); HRMS (MALDI): $m / z$ calcd for $\left[\mathrm{C}_{77} \mathrm{H}_{72} \mathrm{~N}_{4} \mathrm{O}_{4}\right]\left[\mathrm{M}^{+}\right]: 1116.5554$, found 1116.5548 .

\section{5-(3-(Prop-2-yn-1-yloxyphenyl)-10,15,20-tris[3-(4-tert-}

butylbenzyloxy)phenyl]porphyrin 21: The compound was synthesized using general procedure B: a $50 \mathrm{~mL}$ round bottom flask containing $10 \mathrm{~mL}$ of DMF, $2(100 \mathrm{mg}, 0.15$ $\mathrm{mmol}), \mathrm{NaH}(3.5 \mathrm{mg}, 0.15 \mathrm{mmol})$ and propargyl bromide $(0.075 \mathrm{ml}, 0.15 \mathrm{mmol})$ was allowed to stir for $2 \mathrm{~h}$. Yield: $161 \mathrm{mg}(0.14 \mathrm{mmol}, 95 \%)$; m.p. $>300{ }^{\circ} \mathrm{C} ; R_{f}=0.93$ $\left(\mathrm{CH}_{2} \mathrm{Cl}_{2} / n\right.$-hexane/MeOH $\left.=3: 1: 0.2, \mathrm{v} / \mathrm{v} / \mathrm{v}\right) ;{ }^{1} \mathrm{H}$ NMR $\left(400 \mathrm{MHz}, \mathrm{CDCl}_{3}, 25{ }^{\circ} \mathrm{C}\right): \delta_{\mathrm{H}}=$. $2.80(\mathrm{~s}, 2 \mathrm{H}, \mathrm{N} H), 1.33(\mathrm{~s}, 27 \mathrm{H}, t-\mathrm{Bu}-H), 2.6(\mathrm{~s}, 1 \mathrm{H}$, alkyne $), 4.89\left(\mathrm{~s}, 2 \mathrm{H},-\mathrm{CH}_{2}\right), 5.22(\mathrm{~s}$ $\left.6 \mathrm{H}, \mathrm{CH}_{2}\right), 7.44(\mathrm{~m}, 16 \mathrm{H}, \mathrm{Ar}-H / \mathrm{tBu}-\mathrm{Ar}-H), 7.65\left(\mathrm{t},{ }^{3} \mathrm{~J}_{\mathrm{H}-\mathrm{H}}=8.36 \mathrm{~Hz}, 4 \mathrm{H}, \mathrm{Ar}-H\right), 7.81(\mathrm{~d}$, $\left.{ }^{3} J_{\mathrm{H}-\mathrm{H}}=7.12 \mathrm{~Hz}, 3 \mathrm{H}, \mathrm{Ar}-H\right), 7.87(\mathrm{~s}, 5 \mathrm{H}, \mathrm{Ar}-H), 8.86 \mathrm{ppm}\left(\mathrm{d},{ }^{3} J_{\mathrm{H}-\mathrm{H}}=9.68 \mathrm{~Hz}, 8 \mathrm{H}, H_{\beta}\right)$; ${ }^{13} \mathrm{C}$ NMR $\left(100 \mathrm{MHz}, \mathrm{CDCl}_{3} 25^{\circ} \mathrm{C}\right): \delta=23.9,31.4,34.6,56.1,70.1,75.8,114.6,114.7$ $119.9,121.3,125.6,127.5,127.6,127.9,133.8,143.4,143.5,151.1,156.0,157.2 \mathrm{ppm}$ $\mathrm{UV} / \mathrm{Vis}(\mathrm{EtOAc}): \lambda_{\max }(\lg \varepsilon)=417$ (5.02), $515(3.76), 547(3.50), 590(3.15), 647 \mathrm{~nm}$ (2.99); HRMS (MALDI): $\mathrm{m} / \mathrm{z}$ calcd for $\left[\mathrm{C}_{80} \mathrm{H}_{74} \mathrm{~N}_{4} \mathrm{O}_{4}\right]\left[\mathrm{M}^{+}\right]$: 1154.5710, found 1154.5716 .

5,10,15-Tris[3-(hexyloxy)phenyl]-20-(3-hydroxyphenyl)porphyrin 22: Synthesized via General Procedure $\mathrm{C}$ using 2 (400 mg, $0.59 \mathrm{mmol}), \mathrm{K}_{2} \mathrm{CO}_{3}(815 \mathrm{mg}, 5.9 \mathrm{mmol})$, and 1-iodohexane $(1.4 \mathrm{~mL}, 5.9 \mathrm{mmol})$. The reaction was complete after $7 \mathrm{~h}$. Yield: $313 \mathrm{mg}$ of purple crystals $(0.33 \mathrm{mmol}, 40 \%)$; m.p. $>300{ }^{\circ} \mathrm{C}, R_{f}=0.96\left(\mathrm{CH}_{2} \mathrm{Cl}_{2} / n\right.$-hexane/MeOH $=3: 1: 0.2, \mathrm{v} / \mathrm{v} / \mathrm{v}) ;{ }^{1} \mathrm{H}$ NMR $\left(400 \mathrm{MHz}, \mathrm{CDCl}_{3}, 25^{\circ} \mathrm{C}\right): \delta_{\mathrm{H}}=-2.81(\mathrm{~s}, 2 \mathrm{H}, \mathrm{N} H), 0.88(\mathrm{t}$ $\left.{ }^{3} J_{\mathrm{H}-\mathrm{H}}=6.35 \mathrm{~Hz}, 9 \mathrm{H}, \mathrm{CH}\right), 1.16\left(\mathrm{t},{ }^{3} J_{\mathrm{H}-\mathrm{H}}=7.32 \mathrm{~Hz}, 14 \mathrm{H},-\mathrm{CH}_{2}\right), 2.81\left(\mathrm{q},{ }^{3} J_{\mathrm{H}-\mathrm{H}}=7.32 \mathrm{~Hz}\right.$, $\left.10 \mathrm{H},-\mathrm{CH}_{2}\right), 4.14\left(\mathrm{t},{ }^{3} J_{\mathrm{H}-\mathrm{H}}=6.41 \mathrm{~Hz}, 6 \mathrm{H}, \mathrm{OCH}_{2}\right), 7.31\left(\mathrm{~d},{ }^{3} J_{\mathrm{H}-\mathrm{H}}=8.49 \mathrm{~Hz}, 3 \mathrm{H}, \mathrm{Ar}-H\right)$ $7.53\left(\mathrm{t},{ }^{3} J_{\mathrm{H}-\mathrm{H}}=7.91 \mathrm{~Hz}, 1 \mathrm{H}, \mathrm{Ar}-H\right), 7.61\left(\mathrm{t},{ }^{3} J_{\mathrm{H}-\mathrm{H}}=7.91 \mathrm{~Hz}, 3 \mathrm{H}, \mathrm{Ar}-H\right), 7.70\left(\mathrm{~d},{ }^{3} J_{\mathrm{H}-\mathrm{H}}=\right.$ $7.91 \mathrm{~Hz}, 1 \mathrm{H}, \mathrm{Ar}-H), 7.77\left(\mathrm{t},{ }^{3} J_{\mathrm{H}-\mathrm{H}}=8.49 \mathrm{~Hz}, 6 \mathrm{H}, \mathrm{Ar}-H\right), 8.89 \mathrm{ppm}\left(\mathrm{m},{ }^{3} J_{\mathrm{H}-\mathrm{H}}=3.91 \mathrm{~Hz}\right.$, $8 \mathrm{H}, H_{\beta},{ }^{13} \mathrm{C}$ NMR $\left(100 \mathrm{MHz}, \mathrm{CDCl}_{3}, 25{ }^{\circ} \mathrm{C}\right): \delta=14.3,22.4,25.6,29.1,31.4,68.1$, $114.7,115.5,120.0,120.1,120.4,120.41,121.2,122.3,126.2,127.5,128.3,142.8$ 142.9, 156.2, $157.5 \mathrm{ppm}$; UV/Vis (EtOAc): $\lambda_{\max }(\lg \varepsilon)=417$ (5.03), 515 (3.73), 546 (3.50), 591 (3.10), $647 \mathrm{~nm}$ (2.98); HRMS (MALDI): $m / z$ calcd for $\left[\mathrm{C}_{62} \mathrm{H}_{66} \mathrm{~N}_{4} \mathrm{O}_{4}\right]\left[\mathrm{M}^{+}\right]$: 930.5084 , found 930.5080

5,10,15,20-Tetrakis[3-(4-bromobutoxy)phenyl]porphyrin 23: Synthesized via General Procedure $\mathrm{C}$ using $2(400 \mathrm{mg}, 0.59 \mathrm{mmol}), \mathrm{K}_{2} \mathrm{CO}_{3}(815 \mathrm{mg}, 6 \mathrm{mmol})$, and $1,4-$ dibromobutane $(1.4 \mathrm{~mL}, 12 \mathrm{mmol})$. The reaction was complete after 5 h. Yield: $688 \mathrm{mg}$ of purple crystals $(0.57 \mathrm{mmol}, 96 \%)$; m.p. $>300{ }^{\circ} \mathrm{C} ; R_{f}=0.9\left(\mathrm{CH}_{2} \mathrm{Cl}_{2} / n\right.$-hexane $/ \mathrm{MeOH}$ $=3: 1: 0.2, \mathrm{v} / \mathrm{v} / \mathrm{v}) ;{ }^{1} \mathrm{H}$ NMR $\left(400 \mathrm{MHz}, \mathrm{CDCl}_{3}, 25^{\circ} \mathrm{C}\right): \delta_{\mathrm{H}}=-2.67(\mathrm{~s}, 2 \mathrm{H}, \mathrm{N} H), 2.03(\mathrm{~m}$, $\left.8 \mathrm{H},-\mathrm{CH}_{2}\right), 2.14\left(\mathrm{~m}, 8 \mathrm{H},-\mathrm{CH}_{2}\right), 3.52\left(\mathrm{t},{ }^{3} \mathrm{~J}_{\mathrm{H}-\mathrm{H}}=6.16 \mathrm{~Hz}, 8 \mathrm{H},-\mathrm{CH}_{2}\right), 4.19\left(\mathrm{t},{ }^{3} \mathrm{~J}_{\mathrm{H}-\mathrm{H}}=5 \mathrm{~Hz}\right.$ $\left.8 \mathrm{H},-\mathrm{CH}_{2}\right), 7.32\left(\mathrm{~d},{ }^{3} \mathrm{~J}_{\mathrm{H}-\mathrm{H}}=8 \mathrm{~Hz}, 4 \mathrm{H}, \mathrm{Ar}-\mathrm{H}\right), 7.67\left(\mathrm{t},{ }^{3} \mathrm{~J}_{\mathrm{H}-\mathrm{H}}=5 \mathrm{~Hz}, 4 \mathrm{H}, \mathrm{Ar}-\mathrm{CH}_{2}\right), 7.86(\mathrm{~s}$, $\left.4 \mathrm{H}, \mathrm{Ph}-H_{o}\right), 7.90\left(\mathrm{~d},{ }^{3} J_{\mathrm{H}-\mathrm{H}}=7.4 \mathrm{~Hz}, 4 \mathrm{H}, \mathrm{Ar}-\mathrm{CH}_{2}\right), 8.99 \mathrm{ppm}\left(\mathrm{s}, 8 \mathrm{H}, \mathrm{H}_{\beta}\right),{ }^{13} \mathrm{C}$ NMR $(100$ $\left.\mathrm{MHz}, \mathrm{CDCl}_{3}, 25^{\circ} \mathrm{C}\right): \delta=28.0,30.9,32.5,67.1,114.1,119.9,121.2,127.6,127.8$ 143.5, $157.3 \mathrm{ppm}$; UV/Vis (EtOAc): $\lambda_{\max }(\lg \varepsilon)=417$ (5.01), 515 (3.77), 547 (3.52), 590 
(3.15), $647 \mathrm{~nm}$ (2.99); HRMS (MALDI): $m / z$ calcd for $\left[\mathrm{C}_{60} \mathrm{H}_{58} \mathrm{Br}_{4} \mathrm{~N}_{4} \mathrm{O}_{4}\right][\mathrm{M}+]$ : 1214.1192, found 1214.1183 .

[5,10,15,20-Tetrakis(3-hydroxyphenyl)porphyrinato]cobalt(II) 24: Synthesized according to the literature procedure ${ }^{[18]} 100 \mathrm{mg}$ of 2 and cobalt(II)acetate (3 eq.) were according to the literature procedure. $100 \mathrm{mg}$ of 2 and cobalt(II)acetate $(3 \mathrm{eq}$.) were
added to a $100 \mathrm{~mL}$ Erlenmeyer flask containing $10 \mathrm{~mL}$ of DMF. The flask was placed in the microwave and the reaction was run in $3 \times 1$ min intervals at $300 \mathrm{~W}$ with allocated time for cooling of the reaction mixture during each interval. The reaction mixture was taken up into DCM and washed with water $(3 \times 100 \mathrm{~mL})$ to remove residual DMF. The solvent was removed in vacuo and the product purified by recrystallization to yield 106 mg orange/purple solid $24(0.14 \mathrm{mmol}, 98 \%)$; m.p. $>300{ }^{\circ} \mathrm{C} ; R_{f}=0.42\left(\mathrm{CH}_{2} \mathrm{Cl}_{2} / n\right.$ hexane $/ \mathrm{MeOH}=3: 1: 0.2, \mathrm{v} / \mathrm{v} / \mathrm{v}) ;{ }^{1} \mathrm{H}$ NMR $\left(400 \mathrm{MHz},\left(\mathrm{CD}_{3}\right)_{2} \mathrm{SO}, 25^{\circ} \mathrm{C}\right): \delta_{\mathrm{H}}=7.13(\mathrm{~d}$, $\left.{ }^{3} J_{\mathrm{H}-\mathrm{H}}=7.95 \mathrm{~Hz}, 4 \mathrm{H}, \operatorname{Ar}-H\right), 7.36(\mathrm{~s}, 4 \mathrm{H}, \mathrm{o}-\mathrm{Ar}-H), 7.40\left(\mathrm{~d},{ }^{3} J_{\mathrm{H}-\mathrm{H}}=7.32 \mathrm{~Hz} 4 \mathrm{H}, \mathrm{Ar}-H\right)$, $7.49\left(\mathrm{t},{ }^{3} J_{\mathrm{H}-\mathrm{H}}=7.95 \mathrm{~Hz}, 4 \mathrm{H}, m-\mathrm{Ar}-H\right), 8.75 \mathrm{ppm}\left(\mathrm{s}, 8 \mathrm{H}, H_{\beta}\right), 9.81(\mathrm{~s}, \mathrm{br}, 4 \mathrm{H},-\mathrm{OH}) ;{ }^{13} \mathrm{C}$ $\operatorname{NMR}\left(100 \mathrm{MHz},\left(\mathrm{CD}_{3}\right)_{2} \mathrm{SO}, 25^{\circ} \mathrm{C}\right): \delta=115.5,119.3,121.3,125.3,128.5,129.3,130.6$, 132.2, 132.8, 141.6, 142.3, $156.4 \mathrm{ppm}$; UV/Vis (EtOAc): $\lambda_{\max }(\lg \varepsilon)=411(6.24), 525$ $\mathrm{nm}$ (5.09); HRMS (MALDI): $\mathrm{m} / z$ calcd for $\left[\mathrm{C}_{44} \mathrm{H}_{28} \mathrm{CoN}_{4} \mathrm{O}_{4}\right]\left[\mathrm{M}^{+}\right]: 735.1443$, found 735.1435 .

[5,10,15,20-tetrakis(3-oxyphenyl)porphyrin]yltetrabutyl[5,10,15,20-tetrakis(3oxyphenyl)porphyrinato]cobalt(II) $25: \mathrm{K}_{2} \mathrm{CO}_{3}(41 \mathrm{mg}, 0.3 \mathrm{mmol})$ was placed in round bottom flask with $40 \mathrm{~mL}$ of DMF. The flask was fitted with a dropping funne containing a solution of Co- $m$-THPP $(22.4 \mathrm{mg}, 0.03 \mathrm{mmol})$ and $23(40 \mathrm{mg}, 0.03 \mathrm{mmol})$ in $10 \mathrm{~mL}$ of DMF. The solution was added drop-wise over $24 \mathrm{~h}$ and once finished, allowed to stir for a further $12 \mathrm{~h}$. The reaction mixture was diluted with DCM and washed multiple times with water, brine and sodium bicarbonate. The solution was dried of $\mathrm{Mg}_{2} \mathrm{SO}_{4}$ and the solvent removed in vacuo. The resulting orange/purple solid was subjected to column chromatography, with the first orange band being the desired bisporphyrin. Yield: $6.3 \mathrm{mg}$ reddish orange crystals $(0.0038 \mathrm{mmol}, 12 \%)$; m.p. $>300{ }^{\circ} \mathrm{C}$; $R_{f}=0.85\left(\mathrm{CH}_{2} \mathrm{Cl}_{2} / n\right.$-hexane $\left./ \mathrm{MeOH}=3: 1: 0.2, \mathrm{v} / \mathrm{v} / \mathrm{v}\right) ;{ }^{1} \mathrm{H} \mathrm{NMR}\left(600 \mathrm{MHz}, \mathrm{CDCl}_{3}, 25\right.$ $\left.{ }^{\circ} \mathrm{C}\right): \delta_{\mathrm{H}}=-2.77(\mathrm{~s}, 2 \mathrm{H}, \mathrm{NH}), 2.06\left(\mathrm{~m}, 8 \mathrm{H},-\mathrm{CH}_{2}\right), 2.16\left(\mathrm{~m}, 10 \mathrm{H},-\mathrm{CH}_{2}\right), 3.55(\mathrm{~m}, 6 \mathrm{H}$, $\left.\mathrm{CH}_{2}\right), 4.22\left(\mathrm{t}, 8 \mathrm{H},-\mathrm{CH}_{2}\right), 7.34\left(\mathrm{~d},{ }^{3} \mathrm{~J}_{\mathrm{H}-\mathrm{H}}=7.62 \mathrm{~Hz}, 8 \mathrm{H}, \mathrm{Ar}-\mathrm{H}\right), 7.66\left(\mathrm{t},{ }^{3} J_{\mathrm{H}-\mathrm{H}}=7.62 \mathrm{~Hz}\right.$, $8 \mathrm{H}, \mathrm{Ar}-H), 7.79(\mathrm{~s}, 8 \mathrm{H}, \mathrm{Ar}-H), 7.84\left(\mathrm{~d},{ }^{3} J_{\mathrm{H}-\mathrm{H}}=7.62 \mathrm{~Hz}, 8 \mathrm{H}, \mathrm{Ar}-H\right), 8.91 \mathrm{ppm}(\mathrm{m}, 16 \mathrm{H}$ $\left.H_{\beta}\right) ;{ }^{13} \mathrm{C}$ NMR $\left(100 \mathrm{MHz}, \mathrm{CDCl}_{3}, 25{ }^{\circ} \mathrm{C}\right): \delta=27.9,29.4,33.3,66.9,113.9,119.7$, $120.9,127.3,127.6,143.3,157.1 \mathrm{ppm}$; UV/Vis (EtOAc): $\lambda_{\max }(\lg \varepsilon)=417(5.04), 515$ (3.75), 547 (3.50), 589 (3.09), $648 \mathrm{~nm}$ (2.96); HRMS (MALDI): $m / z$ calcd for $\left[\mathrm{C}_{104} \mathrm{H}_{84} \mathrm{CoN}_{8} \mathrm{O}_{8}\right]\left[\mathrm{M}^{+}\right]: 1629.5588$, found 1629.5509 .

\section{Acknowledgements}

This work was supported by a grant from Science Foundation Ireland (P.I 09/IN.1/B2650).

[1] a) P. Rothemund, $J . A m$. Chem. Soc 1936, 57, 625-627; b) P. M. Rothemund, A. R. Menotti, J. Am. Chem. Soc. 1941, 63, 267-270; c) A. D. Adler, F. R. Longo, J. D. Finarelli, J. Goldmacher, J. Assour, L. Korsakoff, J. Org. Chem. 1964, 32, 476.

[2] a) T. J. Dougherty, C. J. Gomer, B. W. Henderson, G. Jori, D. Kessel, M Korbelik, J. Moan, Q. Peng, J. Natl. Cancer Inst 1998, 90, 889-905; b) D. Dolmans, D. Fukumura, R. K. Jain, Nat. Rev. Cancer 2003, 3, 380-387.

[3] a) E. Cariati, M. Pizzotti, D. Roberto, F. Tessore, R. Ugo, Coord. Chem Rev. 2006, 250, 1210-1233; b) A. D. Tillekaratne, R. M. de Silva, K. M. N. de Silva, J. Mol. Struct. 2003, 638, 169-176; c) M. O. Senge, M. Fazekas, M. Pintea, M. Zawadzka, W. J. Blau, Eur. J. Org. Chem. 2011, 5797-5816; d) M. Zawadzka, J. Wang, W. J. Blau, M. O. Senge, J. Phys. Chem. A 2013, 117, 15-26; e) M. O. Senge, M. Fazekas, E. G. A. Notaras, W. J. Blau, M. Zawadzka, O. B. Locos, E. M. N. Mhuircheartaigh, Adv. Mater. 2007, 19, 2737-2774.

[4] a) C. J. Chang, Y. Q. Deng, C. N. Shi, C. K. Chang, F. C. Anson, D. G. Nocera, Chem. Commun. 2000, 1355-1356; b) B. Oregan, M. Grätzel, Nature 1991, 353, 737-740; M. Grätzel, Nature 2001, 414, 338-344.

[5] a) A. Satake, Y. Kobuke, Org. Biomol. Chem. 2007, 5, 1679-1691; b) F. D'Souza, E. Maligaspe, K. Ohkubo, M. E. Zandler, N. K. Subbaiyan, S. Fukuzumi, J. Am. Chem. Soc. 2009, 131, 8787-8797; c) M. R. Wasielewski, Acc. Chem. Res. 2009, 42, 1910-1921; d) A. Harriman, J. P. Sauvage, Chem. Soc. Rev. 1996, 25, 41-48.

[6] a) J. S. Lindsey, in The Porphyrin Handbook, Vol. 1, (Eds. K. M. Kadish, K. M Smith, R. Guilard), Academic Press, San Diego, 2000, pp. 45-118; b) P. D. Rao, S. Dhanalekshmi, B. J. Littler, J. S. Lindsey, J. Org. Chem. 2000, 65 , 7323-7344; c) J. S. Lindsey, I. C. Schreiman, H. C. Hsu, P. C. Kearney, A. M. Marguerettaz, J. Org. Chem. 1987, 52, 827-836; d) J. S. Lindsey, Acc. Chem. Res. 2010, 43, 300-311. e) D. K. Dogutan, J. S. Lindsey, J. Org. Chem. 2008, $73,6728-6742$
[7] a) M. O. Senge, Y. M. Shaker, M. Pintea, C. Ryppa, S. S. Hatscher, A. Ryan, Y Sergeeva, Eur. J. Org. Chem. 2010, 237-258; b) M. O. Senge, Chem. Commun 2011, 47, 1943-1960.

[8] a) W. M. Sharman, J. E. Van Lier, J. Porphyrins Phthalocyanines 2000, 4, 441453; b) M. O. Senge, Acc. Chem. Res. 2005, 38, 733-743; N. N. Sergeeva, V. L. Pablo, M. O. Senge, J. Organomet. Chem. 2008, 693, 2637-2640; S. Horn, B. Cundell, M. O. Senge, Tetrahedron Lett. 2009, 50, 2562-2565; c) M. O. Senge, C. Ryppa, M. Fazekas, M. Zawadzka, K. Dahms, Chem. Eur. J. 2011, 17, 13562-13573.

[9] a) G. M. Vaz, E. Paszko, A. M. Davies, M. O. Senge, PLOS One 2013, 8 , e70653; b) M. B. Bakar, M. Oelgemöller, M. O. Senge, Tetrahedron 2009, 65, 7064-7078; R. Daly, G. Vaz, A. M. Davies, M. O. Senge, E. M. Scanlan, Chem. Eur. J. 2012, 18, 14671-14679; c) M. Verhille, P. Couleaud, R. Vanderesse, D. Brault, M. Barberi-Heyob, C. Frochot, Curr. Med. Chem. 2010, 17, 3925-3943.

[10] a) R. Sharma, A. Long, J. F. Gilmer, Curr. Med. Chem. 2011, 18, 4029-4052; b) F. Majer, J. J. Salomon, R. Sharma, S. V. Etzbach, M. N. M. Najib, R. Keaveny, A. Long, J. Wang, C. Ehrhardt, J. F. Gilmer, Bioorg. Med. Chem. 2012, 20, 1767-1778.

[11] a) R. Bonnett, R. D. White, U. J. Winfield, M. C. Berenbaum, Biochem. J. 1989, 261, 277-280; b) M. O. Senge, J. C. Brandt, Photochem. Photobiol. 2011, 87 , 1240-1296; c) R. Bonnett, D. J. McGarvey, A. Harriman, E. J. Land, T. G. Truscott, U. J. Winfield, Photochem. Photobiol. 1988, 48, 271-276.

[12] a) R. Huisgen, Angew. Chem. 1963, 2, 565-598; b) L. Rogers, F. Majer, N. N. Sergeeva, E. Paszko, J. F. Gilmer, M. O. Senge, Bioorg. Med. Chem. Lett. 2013, 23, 2495-2499.

[13] R. P. Baudouy, P., Tetrahedron 1989, 45, 2067-2074.

[14] a) Y. Q. Mu, R. A. Gibbs, Tetrahedron Lett. 1995, 36, 5669-5672; b) G. A. Molander, T. Ito, Org. Lett. 2001, 3, 393-396; c) A. F. Littke, C. Y. Dai, G. C. Fu, J. Am. Chem. Soc. 2000, 122, 4020-4028.

[15] F. Paul, J. Patt, J. F. Hartwig, J. Am. Chem. Soc. 1994, 116, 5969-5970; A. S. Guram, S. L. Buchwald, J. Am. Chem. Soc. 1994, 116, 7901-7902.

[16] F. Ullmann, P. Sponagel, Ber. Dtsch. Chem. Ges. 1905, 38, 2211-2212.

[17] a) J. X. Qiao, P. Y. S. Lam, Synthesis 2011, 829-856; b) D. M. T. Chan, K. L. Monaco, R. H. Li, D. Bonne, C. G. Clark, P. Y. S. Lam, Tetrahedron Lett. 2003 44, 3863-3865

[18] B. F. O. Nascimento, M. Pineiro, A. M. d. A. R. Gonsalves, M. R. Silva, A. M. Beja, J. A. Paixao, J. Porphyrins Phthalocyanines 2007, 11, 77-84.

[19] Based on HRMS and ${ }^{1} \mathrm{H}$ NMR data.

[20] a) J. P. Collman, J. I. Brauman, T. R. Halbert, K. S. Suslick, Proc. Natl. Acad. Sci. USA 1976, 73, 3333-3337; b) J. P. Collman, R. R. Gagne, C. A. Reed, T. R. Halbert, G. Lang, W. T. Robinson, J. Am. Chem. Soc. 1975, 97, 1427-1439; c) J. P. Collman, R. R. Gagne, T. R. Halbert, J. C. Marchon, C. A. Reed, J. Am. Chem. Soc. 1973, 95, 7868-7870.

[21] S. Smeets, H. Roex, W. Dehaen, Arkivoc, 2003, 83, 83-92.

[22] a) J. P. Collman, P. Denisevich, Y. Konai, M. Marrocco, C. Koval, F. C. Anson, J. Am. Chem. Soc. 1980, 102, 6027-6036; b) J. P. Collman, P. S. Wagenknecht, J. E. Hutchison, Angew. Chem. Int. Ed. Engl. 1994, 33, 1537-1554.

[23] a) M. O. Senge, W. W. Kalisch, K. Ruhlandt-Senge, Chem. Commun. 1996 2149-2150; b) P. D. Harvey, C. Stern, C. P. Gros, R. Guilard, Coord. Chem. Rev. 2007, 251, 401-428.

[24] J. Zhang, Y. Li, W. Yang, S. W. Lai, C. Zhou, H. Liu, C. M Che, Y. Li, Chem. Commun. 2012, 48, 3602-3604

[25] The compound appears as the second fraction in column chromatography, eluent after unreacted starting material 24. ${ }^{1} \mathrm{H}$ NMR analysis proved difficult due to the presence of the cobalt center, however, confirmation of the structure came from HSQC and HMBC analysis along with HRMS confirmation.

[26] A. Ryan, A. Gehrold, R. Perusitti, M. Pintea, M. Fazekas, O. B. Locos, F. Blaikie, M. O. Senge, Eur. J. Org. Chem. 2011, 5817-5844.

[27] Superbase $=2,8,9$-Triisobutyl-2,5,8,9-tetraaza-1-phosphabicyclo[3.3.3]undecane.

[28] The di- and trisubstituted derivatives were observed on TLC and confirmed by HRMS, however, their synthesis and isolation were not optimized within this current project.

Received: ((will be filled in by the editorial staff)) Revised: ((will be filled in by the editorial staff)) Published online: ((will be filled in by the editorial staff)) 
Entry for the Table of Contents (Please choose one layout only)

Layout 1:

Porphyrin Synthesis

Luke Rogers, Emeralda Burke-

Murphy, Mathias O. Senge*

............. Page - Page

Simple Porphyrin

Desymmetrization: $m$ THPP as a

Gateway Molecule for Peripheral

Functionalization
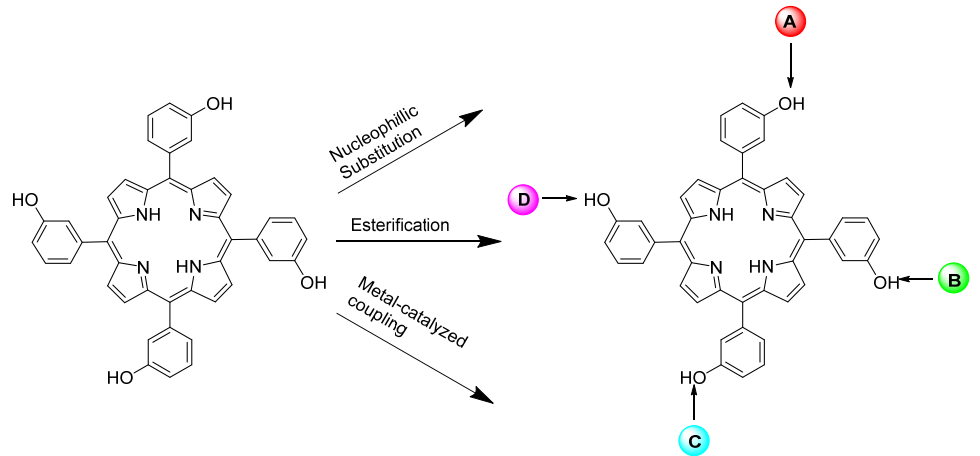